\title{
Intelligent Reflecting Surface Assisted Beam Index-Modulation for Millimeter Wave Communication
}

\author{
Sarath Gopi and Sheetal Kalyani and Lajos Hanzo
}

\begin{abstract}
Millimeter wave communication is eminently suitable for high-rate wireless systems, which may be beneficially amalgamated with intelligent reflecting surfaces (IRS), while relying on beam-index modulation. Explicitly, we propose three different architectures based on IRSs for beam-index modulation in millimeter wave communication. Our schemes are capable of eliminating the detrimental line-of-sight blockage of millimeter wave frequencies.The schemes are termed as single-symbol beam index modulation, multi-symbol beam-index modulation and maximum-SNR single-symbol beam index modulation. The principle behind these is to embed the information both in classic QAM/PSK symbols and in the transmitter beam-pattern. Explicitly, we proposed to use a twin-IRS structure to construct a low-cost beamindex modulation scheme. We conceive both the optimal maximum likelihood detector and a low-complexity compressed sensing detector for the proposed schemes. Finally, the schemes designed are evaluated through extensive simulations and the results are compared to our analytical bounds.
\end{abstract}

Index Terms-Intelligent reflecting surfaces, beam index modulation, phased array antenna, compressed sensing.

\section{INTRODUCTION}

Next-generation systems are expected to satisfy substantially improved specifications. Furthermore, new solutions, such as the Internet of Things (IoT), massive machine type communications (MTC) also contribute to the escalating mobile data traffic, as predicted by the International Telecommunication Union (ITU) [1]. Hence researchers aim for increasing the degrees of design-freedom in support of these ambitious requirements.

The $30-300 \mathrm{GHz}$ so-called millimeter wave (mmWave) frequency band has substantial hitherto unexploited bandwidth resources for supporting Gigabit per seconds $(\mathrm{Gb} / \mathrm{s})$ data rates [2]-[4]. For example, in indoor scenarios a data rate of upto $6.7 \mathrm{Gbps}$ is

Sarath Gopi and Sheetal Kalyani are with the Dept. of Electrical Engineering, Indian Institute of Technology, Madras, India (Emails: \{ee14d007@ee, skalyani@ee\}.iitm.ac.in).

Lajos Hanzo is with the School of Electronics and Computer Science, University of Southampton.(Email: hanzo@soton.ac.uk).

L. Hanzo would like to acknowledge the financial support of the Engineering and Physical Sciences Research Council projects EP/N004558/1, EP/P034284/1, EP/P034284/1, EP/P003990/1 (COALESCE), of the Royal Society's Global Challenges Research Fund Grant as well as of the European Research Council's Advanced Fellow Grant QuantCom. achieved by the IEEE 802.11ad standard developed at $60 \mathrm{GHz}$ frequency [5]. This result has ignited research interest in this frequency range also for outdoor scenarios. In an early experiment, it has been shown that mmWave communication is capable of achieving a peak data rate of $1 \mathrm{Gbps}$ in an outdoor environment for a communication range of upto $1.7 \mathrm{~km}$ at moderate Bit Error Rates (BERs) [6]. This system used only $500 \mathrm{MHz}$ of bandwidth at $28 \mathrm{GHz}$. Naturally, there are a number of propagation challenges to be overcome, since typically only line-of-sight (LOS) communication is possible at these frequencies, which also suffer from fading, significant absorption losses in the atmosphere and building-penetration losses [7] [8].

Furthermore, researchers are also aiming for reducing both the power consumption and hardware cost. Intelligent Reflecting Surfaces (IRS) offer a viable solution for meeting these requirements [9]. Explicitly, IRSs constitute passive reflecting surfaces equipped with integrated electronic circuits, which are capable of imposing carefully controlled amplitude and/or phase shifts on the incident signals [10]-[12]. The concept has been earlier proposed in [13] and its employment as a phase-shifter has become popularized by [14]. IRSs are eminently suitable for energy-efficient solutions in a wide variety of applications, such as signal-to-noise-ratio (SNR) maximization [15], ratemaximization [16], [17], for improving the energy efficiency [18], [19], for minimizing transmit power [20], for providing secure communication [21], multi-cell MIMO communication [22], [23], over the air computation [24], low latency mobile edge computing [25], index modulation [26] and so on.

In [27], analog beamforming based beam-index modulation has been proposed as an extension of spatial modulation [28], [29]. Inspired by these results, we conceive IRS assisted beam-index modulation for mmWave communications. Beamforming techniques have been exploited in mmWave communication for mitigating their path loss [30]-[32], for achieving directional transmission [33]-[35], for avoiding intercarrier-interference [36] and also for safeguarding against eavesdroppers [37]. However, there is a paucity of contributions on beamforming-aided index modulation in IRS-assisted mmWave communication. Our main contributions are: 


\begin{tabular}{|l|l|l|l|l|l|l|l|}
\hline & Our Scheme & {$[27]-2018$} & {$[15]-2019$} & {$[38]-2020$} & {$[26]-2020$} & [39] -2020 & [40]-2020 \\
\hline Multi IRS assisted multihop & $\checkmark$ & & & & & $\checkmark$ \\
\hline mmWave & $\checkmark$ & & & & & $\checkmark$ & \\
\hline non-LOS & $\checkmark$ & $\checkmark$ & & $\checkmark$ & $\checkmark$ & $\checkmark$ & \\
\hline Beam Index Modulation & $\checkmark$ & $\checkmark$ & & & & \\
\hline SNR Optimization & $\checkmark$ & & $\checkmark$ & $\checkmark$ & $\checkmark$ & & \\
\hline Beamformer Gain & $\checkmark$ & & & & $\checkmark$ & \\
\hline Rician Channel Model & $\checkmark$ & & & & $\checkmark$ \\
\hline $\begin{array}{l}\text { BER analysis based on } \\
\text { Non-Gaussian Approximation }\end{array}$ & $\checkmark$ & & & & \\
\hline
\end{tabular}

COMPARISON OF THE PROPOSED SCHEME WITH SIMILAR IDEAS.

1) We propose IRS assisted beam index modulation for mmWave communication. Beamforming solutions proposed for mmWave frequencies tend to rely on either analog beamforming [41], [42] or on hybrid techniques [6], [39], [43]-[48]. In [49] digital beamforming is proposed, which relies on complex hardware. As a remedy, IRS has been proposed for imposing phase shifts on the incident signal, which can be exploited for beamforming. As a further benefit, they are capable of circumventing the predominantly LOS nature of mmWave propagation. Hence, our proposed scheme has at least three appealing features: it supports non-LOS communication at mmWave frequencies at a low cost, whilst conveying extra information via beam-index modulation.

2) We propose three different architectures for IRS assisted beam-index modulation. The first is termed as single-symbol beam index modulation, where the information is carried both by classic QAM/PSK symbols and by the transmitter beampattern. This idea has also been extended for further improving the data rate in our Scheme 2, which is a multi-symbol beam-index modulation arrangement. In the third scheme, we provide an architecture for improving the SNR of the proposed beam-index modulation.

3) The optimal maximum likelihood (ML) detector is derived for the schemes conceived. Additionally, a low complexity compressed sensing assisted detector is also developed.

4) An upper bound of the average BER is obtained for the optimal ML detector. Finally, the proposed scheme is evaluated through extensive simulations and its performance is compared to the theoretically obtained bound.

In Table I, we provide a bold summary and contrast our new contributions to the seminal literature. The key contribution of our scheme is a unique twinIRS architecture, in which one of the IRSs can be positioned farther away from the transmitter. IM on this IRS is activated wirelessly using the other IRS. This architecture benefits in terms of accomplishing nonLOS communication by two LOS paths, additionally achieving a substantial beamformer gain and hence an SNR gain. The rest of the paper is organized as follows. Section II details the proposed IRS assisted beam-index modulation schemes. The implementation aspects and parameter design of the schemes are detailed in Section III, while our detectors are developed in Section IV. In Section V, the error analysis of the proposed scheme is provided. Our simulation results are given in Section VI and we conclude in Section VII.

Notations: Throughout the paper, unless otherwise specified, bold lower case and bold upper case letters are used to represent vectors and matrices, respectively. $\mathbf{A}^{H}, \operatorname{Tr}\{\mathbf{A}\}$ and $\lambda_{\text {min }}(\mathbf{A})$ represents the hermitian, trace and minimum eigen value of $\mathbf{A}$, respectively. $\|$.$\| stands$ for $L_{2}$ - norm. $|a|$ and $\operatorname{Re}\{a\}$ is the absolute and real value of scalar $a$, respectively. $\mathbf{I}$ is the identity matrix of appropriate dimension. $C \mathcal{N}(\mu, \mathbf{C})$ represents the complex Gaussian distribution with mean vector $\mu$ and covariance matrix C. $\lfloor b\rfloor$ is the largest integer not greater than $b$. $\Gamma($.$) is the \Gamma$-function, i.e., $\Gamma(z)=\int_{0}^{\infty} x^{z-1} e^{-x} d x$ and for integer $z, \Gamma(z)=(z-1)$ ! and $\Gamma(a, b)$ is the Gamma distribution with $a$ and $b$ are shape and rate parameters, respectively.

\section{PRoposed IRS Assisted BeAM-INDEX MODULATION SCHEMES}

We propose three different IRS assisted beam-index modulation schemes. All these schemes are single input multiple output (SIMO) arrangements, containing a transmitter antenna (TA), two sets of IRSs and $N_{R}$ receiver antennas (RAs). Each IRS has one or more reflecting surfaces (RS) and each RS has many elements. The first IRS, namely $I R S_{1}$, can be directly accessed by the transmitter and it is used for selectively activating the elements in the second IRS, i.e. in $I R S_{2}$. The block diagram of the proposed scheme is sketched in Fig. 1, which is further elaborated on using Fig. 2. The steps from (1) to (5) in the Fig. 2 is detailed below.

1) The incoming bit sequence is split into two groups. The first group is used for selecting the classic PSK/QAM symbols, while the second set is used for beam-index modulation.

2) The TA and $I R S_{1}$ are kept close to each other. They have both wired and wireless connections. Based on the first group of bits, an appropriate PSK/QAM symbol $(s)$ is selected at the transmitter, which is transmitted wirelessly to each RS in $I R S_{1}$. 


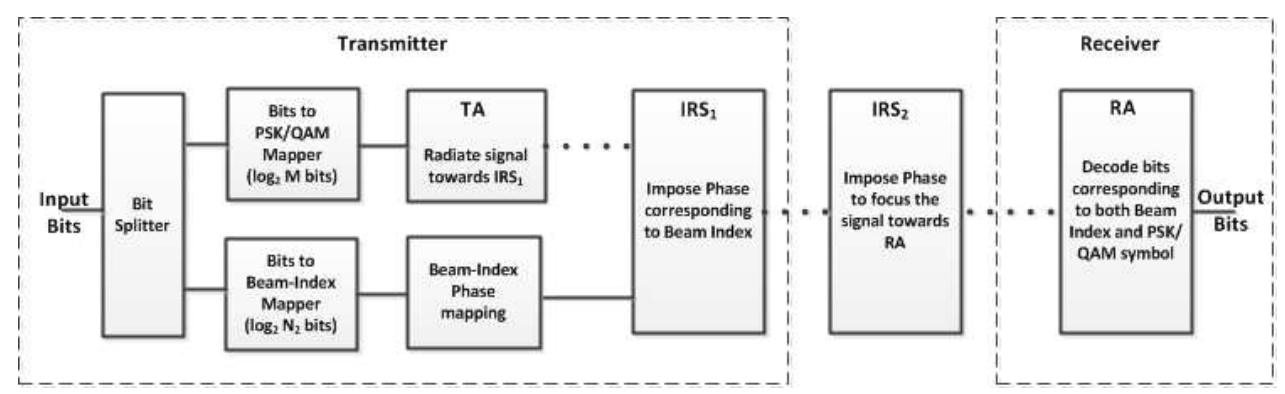

Fig. 1. Proposed IRS assisted beam-index modulation scheme. Solid and dotted lines indicate wired and wireless links, respectively.

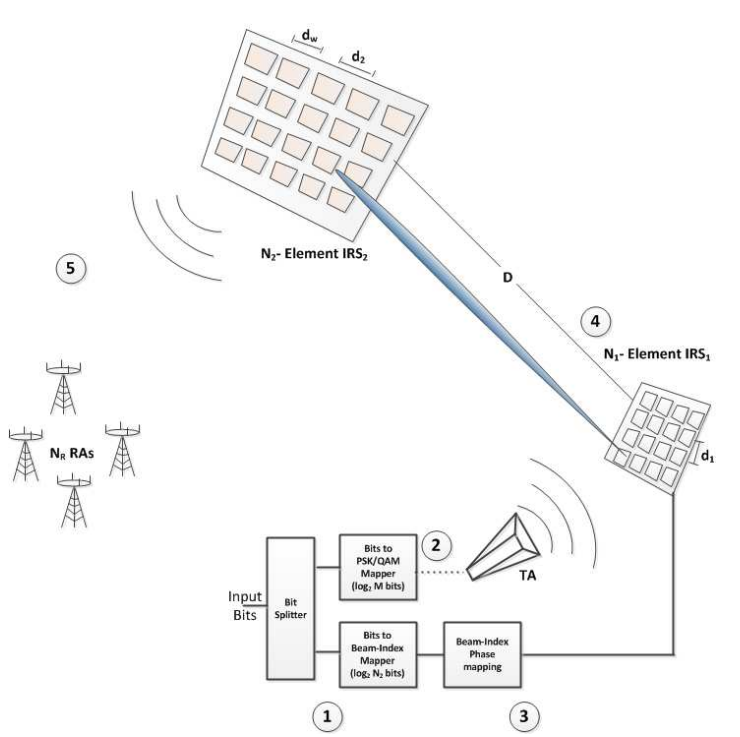

Fig. 2. Architecture of single-symbol beam-index modulation.

3) The wired connection is used for mapping the second group of bits onto beam-index modulation. These bits are converted to the appropriate phase vector, which are then forwarded to the elements of the RSs in $I R S_{1}$.

4) Based on the received phase vector, the elements in $I R S_{1}$ impose the required phase shift on the incident signal, which are then forwarded to $I R S_{2}$. The phase is specifically adjusted for ensuring that only the desired elements in $I R S_{2}$ receive the signal. This specific selection is determined based on the information bits reserved for beam-index modulation.

5) Each element of $I R S_{2}$ induce a constant phase to reflect the signal towards the RAs. This is captured by the RAs. The information detected at the RAs includes both the conventional PSK/QAM symbols and the specific element indices of $I R S_{2}$, which reflect the symbols. This is done jointly by $N_{R}$ RAs.

For detailing the schemes, we will make the following assumptions.

1) The channel between the TA and $I R S_{1}$ may be deemed to be a low-noise AWGN channel. A conventional horn antenna having a few centimetre length can be used as the TA, where $I R S_{1}$ is positioned, say 4-5 $\mathrm{m}$ away from the TA [50]. Hence, the distance should satisfy the far field condition, albeit this is not actually necessary, since the position of the TA is perfectly known at $I R S_{1}$, hence near-perfect delay compensation can be arranged for this location. The TA is designed in such a way that the signal is pointed exactly towards $I R S_{1}$. Finally, $I R S_{1}$, which is a passive device, introduces a phase shift and reflects the signal towards $I R S_{2}$. Hence, the only source of noise, that can affect the signal is the one, which is added at $I R S_{1}$, and this is negligible.

2) There is only LOS communication between $I R S_{1}$ and $I R S_{2}$. Typically, a Rician channel model is used for modelling IRS-assisted communication systems [51]. However, for the proposed scheme the elements in $I R S_{1}$ adjust the phase in such a way that it forms a directional beam and only the specifically selected elements of $I R S_{2}$ receive the signal. Moreover, $I R S_{2}$, which is close to $I R S_{1}$, is carefully positioned for ensuring that there is no blockage between $I R S_{1}$ and $I R S_{2}$. Hence, the channel between $I R S_{1}$ and $I R S_{2}$ is assumed to be an AWGN channel.

3) Between $I R S_{2}$ and the receiver, we have a Rician channel model.

A beneficial application of the proposed architectures can be found in the Internet of Things(IoT), where the desired information has to be collected by sensors and delivered to either a distant server or to a user. The various applications include smart homes, industrial and environmental monitoring, building and home automation etc. Let us assume that the information collected from a home or an industrial cite should be communicated to a $\mathrm{BS}$, from where the information can be communicated to the destination through the cellular network. In this case, the TA and $I R S_{1}$, which only belong to the specific user, can be placed in the terrace of the home or at the industrial cite. The $I R S_{2}$ can be situated at the top of a tall building in the vicinity, which can be shared among many such users, who have orthogonal resources. The details of the schemes are given below. 


\section{A. Scheme 1: Single-Symbol Beam-Index Modulation}

This scheme is shown in Fig. 2. In this scheme, both the IRSs have only a single RS. IRS $S_{1}$ is directly connected to the transmitter, whereas $I R S_{2}$ is kept at a distance, say $D$, from the first IRS. Let $I R S_{1}$ be a $\left(N_{1 H} \times N_{1 W}\right)$ element array, while $I R S_{2}$ be an $\left(N_{2 H} \times N_{2 W}\right)$ array and let $N_{1}=N_{1 H} N_{1 W}$ and $N_{2}=N_{2 H} N_{2 W}$ be the total number of elements in $I R S_{1}$ and $I R S_{2}$, respectively. The TA sends the symbols to $I R S_{1}$, where each element applies a specific phase shift to the incident wave so that only one of the elements in $I R S_{2}$ receives the signal. Hence, in this scheme the total number of bits per channel use (bpcu) is $\log _{2} M+\left\lfloor\log _{2} N_{2}\right\rfloor$, where the first term corresponds to the QAM/PSK symbols, while the second term corresponds to the selection of the element in $I R S_{2}$. Finally, $I R S_{2}$ reflects the signal and it is received at the RAs.

Let $s$ be the transmitted symbol. The symbol received at $I R S_{1}$ is $s+w_{1}$, where $w_{1} \sim \mathcal{C N}\left(0, \sigma_{1}^{2}\right)$. However, under Assumption 1, we have $\sigma_{1}^{2} \approx 0$ and the contribution $w_{1}$ can be discarded. Therefore, the vector received at $I R S_{2}$ is:

$$
\mathbf{x}_{2}=\mathbf{b} s+\mathbf{w}_{2},
$$

where $\mathbf{w}_{2} \sim \mathcal{C N}\left(0, \sigma_{2}^{2}\right)$ under Assumption 2) and b is an $N_{2} \times 1$ vector. Ideally, b should have only a single non-zero entry corresponding to the index of the beam (or equivalently corresponding to the selected element in $I R S_{2}$ ). However, this will not happen in practice, since a finite power will be dispersed on other directions also and this power distribution depends on the beampattern. The vector at the receiver can be written as:

$$
\begin{aligned}
\mathbf{y} & =\mathbf{H} \Theta \mathbf{x}_{2}+\mathbf{w}_{R} \\
& =\mathbf{H} \Theta \mathbf{b} s+\mathbf{w},
\end{aligned}
$$

where $\mathbf{H}$ is the $N_{R} \times N_{2}$ channel matrix as defined under Assumption 3, $\Theta$ is an $\left(N_{2} \times N_{2}\right)$ diagonal matrix of phase shifts given by the elements in $I R S_{2}$ and $\mathbf{w}_{R} \sim \operatorname{CN}\left(0, \sigma_{R}^{2}\right)$. Note that $\mathbf{w}=\mathbf{H} \Theta \mathbf{w}_{2}+\mathbf{w}_{R}$ is the additive noise component having a distribution of $C \mathcal{N}(0, \Sigma)$, where $\Sigma=\mathbf{H H}^{H} \sigma_{2}^{2}+\sigma_{R}^{2} \mathbf{I}$. Finally, the receiver has to detect both $\mathbf{b}$ and $s$ from $\mathbf{y}$ to decode the transmitted bits. The detection schemes will be discussed in Section IV.

This scheme predominantly uses only a fraction of elements of the second reflecting surface, instead of exploiting all of them to improve the attainable beamformer gain. However, it should be noted that most of the transmitted energy is focussed on the intended elements, while the power impinging on all other elements is negligibly small, since beamforming is used in the first stage. Hence, with the aid of the proposed scheme, we will get the dual advantages of both a beneficial beamforming gain and the additional advantage of an increased data rate.

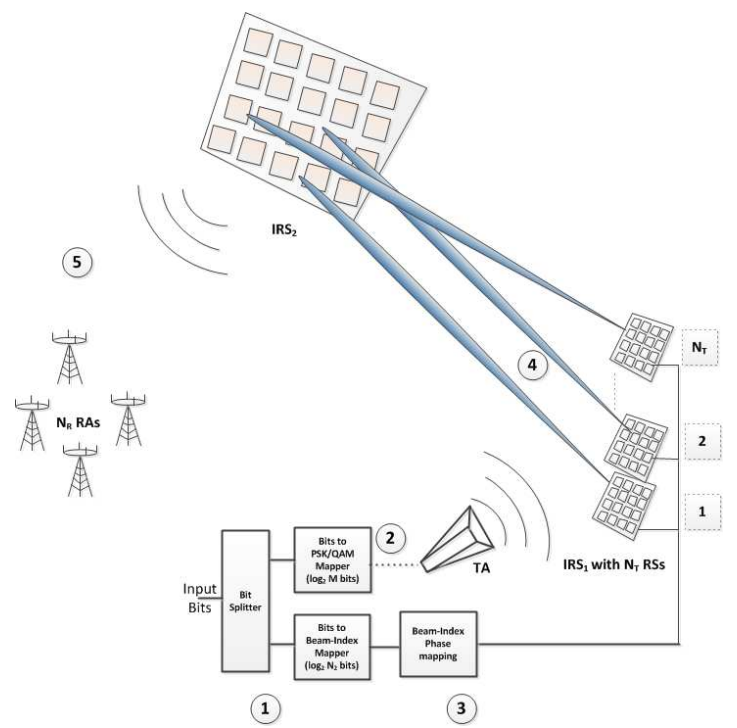

Fig. 3. Architecture of multi-symbol beam-index modulation. Contrast to Fig. 2, there are $N_{T}$ RSs in $I R S_{1}$ in this case.

\section{B. Scheme 2: Multi-Symbol Beam-index Modulation}

In the second scheme, the first scheme is extended to multi-symbol communication. The architecture is shown in Fig. 3. In this case, there are $N_{T} \mathrm{RSs}$ in $I R S_{1}$ contrast to a single RS in Scheme 1. The modulator identifies $N_{T}$ different phase-vectors depending on the bit sequence corresponding to the beam-index modulation and each vector is fed to different RSs in $I R S_{1}$. Therefore, $N_{T}$ RSs focus the conventional QAM/PSK symbol onto $N_{T}$ different elements of $I R S_{2}$. Hence, in this case, the total number of bpcu is $\log _{2} M+\left\lfloor\log _{2}\left(\begin{array}{c}N_{2} \\ N_{T}\end{array}\right) \mid\right.$. Therefore, this scheme provides a higher data rate than scheme 1 . The choice of the elements to be activated can be organized using a look up table method or the combinatoric approach [52], [53].

Mathematically, this scheme can be represented using Equations (1) and (2). However, the difference is that in this case, ideally there will be $N_{T}$ non-zero entries in $\mathbf{b}$.

\section{Scheme 3: Maximum-SNR Single-Symbol Beam- Index Modulation}

Fig. 4 shows the architecture of this scheme. This is similar to Scheme 1, except that in this case each element of $I R S_{2}$ is replaced by an RS having $N_{3}$ elements. Hence, there will be a total of $N_{2} N_{3}$ elements in $I R S_{2}$. Both the TA and $I R S_{1}$ function in the same way as in the case of single-symbol beam-index modulation. Hence, the signal received at $I R S_{2}$ can be written using (1). However, in contrast to the other two cases, here the elements in $I R S_{2}$ apply a phase shift to the incident signal. The phase shift in $I R S_{2}$ is adjusted in such a way that the SNR at the receiver is maximized.

Note that in (2) $\mathbf{H}$ and $\Theta$ is an $\left(N_{R} \times N_{2} N_{3}\right)$ and $\left(N_{2} N_{3} \times N_{2} N_{3}\right)$ matrix, respectively in this case. Fur- 


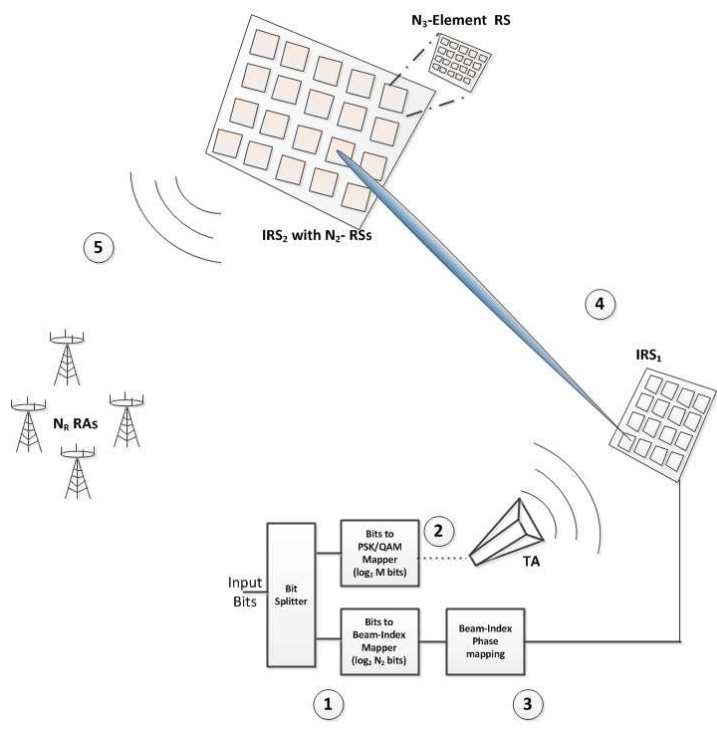

Fig. 4. Architecture of the Maximum-SNR Single-Symbol BeamIndex Modulation. Contrast to Fig. 2, there are $N_{3}$ elements in each RS of $I R S_{2}$, which impose phase shift on the incident signal to maximize SNR at the RAs.

thermore, $\Theta$ is not a constant matrix, but depends on H. The overall SNR in this case is defined as:

$$
\mathrm{SNR}=\frac{\|\mathbf{H} \Theta \mathbf{b} s\|^{2}}{\operatorname{Var}(\|\mathbf{w}\|)}
$$

where the denominator is the variance of the norm of the vector $\mathbf{w}$. The SNR can be maximized by maximizing the numerator of (3), since the denominator is independent of $\Theta$, which is the maximization variable. Let $\theta_{1: N_{2} N_{3}}$ represents the entries of the diagonal of $\Theta$. Hence, the SNR maximization can be written as:

$$
\max _{\theta_{l}}\|\mathbf{H} \Theta \mathbf{b} s\|^{2} \text { s.t. }\left|\theta_{l}\right|=1, \forall l=1,2, \ldots, N_{2} N_{3} .
$$

However, the above optimization problem has the following challenges. $I R S_{2}$ is a passive device and it may not be practical to solve a complex optimization problem there. Hence, the optimization should ideally be carried out at transmitter or receiver and the resultant information has to be communicated to $I R S_{2}$. Therefore, if the optimization depends on the data to be transmitted (bs), $\Theta$ has to be updated in every time slot, which is a substantial communication overhead. Hence, the optimization should preferably only depend on either an average value of $\mathbf{b} s$ or indeed ideally should be independent of it. Accordingly, we will propose the following solutions for (4).

1) Solution 1: This solution is based on the assumption that an ideal beam pattern exists, i.e., all elements in the selected RS of $I R S_{2}$ receives the same power, while all other elements receive no power. Without loss of generality, let this constant be 1 . Hence, the optimization function in (4) can be written as:

$$
\|\mathbf{H} \Theta \mathbf{b} s\|^{2}=\|\mathbf{H} \Theta \mathbf{1}\|^{2}=\|\mathbf{H} \boldsymbol{\theta}\|^{2},
$$

where $\mathbf{1}$ is a vector of $1 \mathrm{~s}$ and $\boldsymbol{\theta}$ is a vector formed from the diagonal elements $\theta_{1: N_{2} N_{3}}$ of $\Theta$. Hence, the maximization problem (4) becomes:

$$
\begin{aligned}
& \max _{\theta_{l}} \boldsymbol{\theta}^{H} \mathbf{H}^{H} \mathbf{H} \boldsymbol{\theta} \\
& \text { s.t. }\left|\theta_{l}\right|=1, \forall l=(\hat{I}-1) N_{3}+1, \ldots, \hat{I} N_{3},
\end{aligned}
$$

where $\hat{I}$ is the specifically selected $\mathrm{RS}$ in $I R S_{2}$. Let $\theta_{l}=e^{j \alpha_{l}}$, since $\left|\theta_{l}\right|=1$. Bearing this in mind and noting that $\mathbf{H}^{H} \mathbf{H}$ is a Hermitian matrix, (4) is reformulated as the following unconstrained optimization problem.

$$
\max _{\alpha_{l}} \sum_{i=(\hat{I}-1) N_{3}+1}^{\hat{I} N_{3}} \sum_{j=(\hat{I}-1) N_{3}+1}^{\hat{I} N_{3}} \mathcal{R} e\left\{e^{j\left(\alpha_{i}-\alpha_{j}\right)}\left(\mathbf{H}^{H} \mathbf{H}\right)_{i j}\right\},
$$

where $\left(\mathbf{H}^{H} \mathbf{H}\right)_{i j}$ is the $(i, j)^{t h}$ element of $\mathbf{H}^{H} \mathbf{H}$. Since (7) is not a concave function, it can only be solved using some iterative technique for finding its local maximum.

2) Solution 2: This solution relies on the assumption that $N_{R} \geq N_{3}$, i.e. there are more number of RAs than the number of elements in the RS of $I R S_{2}$. In order to develop the solution, let us state and prove Lemma 1.

Lemma 1. Let $\mathbf{H}_{Q}$ and $\Theta_{Q}$ be the $\left(N_{R} \times N_{3}\right)$ and $\left(N_{3} \times N_{3}\right)$ sub-matrices of $\mathbf{H}$ and $\Theta$ corresponding to the selected RS, respectively and let $\mathbf{b}_{Q}$ be the corresponding sub-vector of $\mathbf{b}$. If $N_{R} \geq N_{3}$, with probability 1 , the bound

$$
\left\|\mathbf{H}_{Q} \Theta_{Q} \mathbf{b}_{Q} s\right\|^{2} \geq \lambda_{\min }\left(\Theta_{Q}^{H} \mathbf{H}_{Q}^{H} \mathbf{H}_{Q} \Theta_{Q}\right) \operatorname{Tr}\left\{\left(\mathbf{b}_{Q} s\right)\left(\mathbf{b}_{Q} s\right)^{H}\right\}
$$

is non-trivial, which equivalently leads to $\lambda_{\min }\left(\Theta_{Q}^{H} \mathbf{H}_{Q}^{H} \mathbf{H}_{Q} \Theta\right)>0$.

Proof. See Appendix A for proof.

Lemma 1 can be used for solving the optimization problem (4). The idea is to maximize the non-trivial lower bound instead of the actual function. Hence, the optimization problem (4) becomes:

$$
\begin{aligned}
& \max _{\theta_{l}} \lambda_{\min }\left(\Theta_{Q}^{H} \mathbf{H}_{Q}^{H} \mathbf{H}_{Q} \Theta\right) \\
& \text { s.t. }\left|\theta_{l}\right|=1, \forall l=(\hat{I}-1) N_{3}+1, \ldots, \hat{I} N_{3} .
\end{aligned}
$$

We know that $\lambda_{\min }(\mathbf{A})=\min _{\|\mathbf{z}\|=1}\|\mathbf{A z}\|$ [54, Eq. 7.5.4] Therefore (9) can be rewritten as:

$$
\begin{aligned}
& \max _{\theta_{l}} \min _{\mathbf{z}}\left\|\Theta_{Q}^{H} \mathbf{H}_{Q}^{H} \mathbf{H}_{Q} \Theta \mathbf{z}\right\| \\
& \text { s.t. }\|\mathbf{z}\|=1, \quad\left|\theta_{l}\right|=1, \forall l=(\hat{I}-1) N_{3}+1, \ldots, \hat{I} N_{3},
\end{aligned}
$$

where (10) is a constrained non-linear minimax optimization problem. This can be solved directly [55], [56]. Alternatively, it can be converted into a non-linear maximization problem by introducing an additional variable and then solved using standard techniques. 
It should be noted that in both solutions of the SNR maximization problem, $\hat{I}$, i.e. the selected data dependent RS of $I R S_{2}$ that has to be optimized, depends on the information bits. In order to avoid the dependence of optimization on the information bits, each RS is optimized separately whenever there is considerable change in the channel. The optimized phase information is passed to $I R S_{2}$, which applies phase shifts to all elements instead of the selected RS. This scheme can be extended to the case of multisymbol beam-index modulation (Scheme 2), where there will be $N_{T}$ RSs in $I R S_{1}$, which activate $N_{T}$ RSs in $I R S_{2}$. Finally, all the activated RSs in $I R S_{2}$ can apply a phase shifts for improving the SNR. Thus the scheme will have both an improved data rate and improved SNR. Practically, the optimal phase shifts have to be estimated at the receiver and then communicated to $I R S_{2}$ whenever there is significant change in the channel characteristics.

\section{IMPLEMENTATION OF BEAM-INDEX MODULATION}

The principle behind the proposed beam-index modulation is the data-dependent activation of the elements in $I R S_{2}$. This is achieved by appropriately choosing the phase shifts applied by the elements in $I R S_{1}$. In order to estimate the phase shifts, it is assumed that there is only LOS communication between $I R S_{1}$ and $I R S_{2}$. Therefore, the phase shifts only depend on the geometry of the pair of IRSs. The estimation of phase shifts is detailed below.

Let the centre of $I R S_{1}$ be the origin co-ordinate $(0,0,0)$ and $\mathbf{P}$ be the position vector of elements of $I R S_{1}$. Let the $n^{\text {th }}$ element of $I R S_{2}$ be activated by $I R S_{1}$ according to the input bit sequence and let $\left(\phi_{n}^{h}, \phi_{n}^{v}\right)$ represents the azimuth and the elevation angle pair for this element with respect to the origin. Then, the phasevector to be given by the elements of $I R S_{1}$ to choose the $n^{\text {th }}$ beam is $\psi=2 \pi f \tau$, where $\tau=\frac{\mathbf{P}^{H} \mathbf{u}_{n}}{c}$ with $c$ and $f$ being the speed of the light and the carrier frequency,

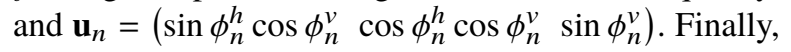
in the case of multi-bit beam-index modulation, these phase shifts have to be calculated for each of IRSs according to the input bit sequences.

Additionally, if the IRS elements can modify the amplitude of the incident signal along with the phase, one can modify the relative weighting of each element. Since IRSs constitute passive devices, amplification may be difficult to achieve and will not be a cost effective solution. However, attenuation can be readily applied to the incident signal [57]. The attenuation can be adjusted in such a way that it acts as a window function for the beamforming and the beam pattern can be accordingly modified. This will help in reducing the interference, which will be discussed in Section V. In Section III-A, the design of two IRSs is detailed.

\section{A. Parameter Design}

The parameters to be designed are the number of elements and the corresponding inter-element spacing in $I R S_{1}$, as well as in $I R S_{2}$ and the distance between two IRSs. Let $\lambda$ be the wavelength corresponding the highest frequency of operation. We will fix the design parameters as follows [58].

1) Inter-element spacing in $I R S_{1}\left(d_{1}\right)$ : The elements in each RSs of $I R S_{1}$ should be spaced at $\frac{\lambda}{2}$ distances. For example, if the maximum operating frequency is $f_{\text {max }}=60 \mathrm{GHz}$, then the spacing between the elements in $I R S_{1}$ is $d_{1}=2.5 \mathrm{~mm}$. Now, if $I R S_{1}$ is a $100 \times 100$ element system, then its dimension is going to be as compact as $0.25 \times 0.25 \mathrm{~m}$. This spacing is important for avoiding grating lobes in the beams formed using $I R S_{1}$ [58, eq. 2.117].

2) Distance between two IRSs $(D)$ : The distance $(D)$ between $I R S_{1}$ and $I R S_{2}$ should meet the far field condition of $D>\frac{2 L^{2}}{\lambda}$, where $L$ is the length of the RS [59, pp. 32]. In the above example $D>25 \mathrm{~m}$. If this condition is met, it can be assumed that the wave front travelling from $I R S_{1}$ to $I R S_{2}$ is planar, so that the phase shifts can be computed as detailed in Section III.

3) Inter element spacing in $I R S_{2}\left(d_{2}\right)$ : The width of each element in $I_{R} S_{2}\left(d_{w}\right)$ should be less than $D \theta_{B W}$, where $\theta_{B W}$ is the beam-width of $I R S_{1}$ and the separation $d_{2}$ between elements in $I R S_{2}$ should be higher than this value. These conditions ensure that the intended element and only the intended element receives the signal reflected by $I R S_{1}$. For a rectangular window, the approximate beam-width is $\theta_{B W} \approx \frac{50 \lambda}{L}$ [58, Eq. (2.100)]. In the example we have considered $\theta_{B W} \approx 1^{0}$ corresponding to the minimum frequency. Therefore $d_{w}<48 \mathrm{~cm}$ and $d_{2}>48 \mathrm{~cm}$ for $D=25 \mathrm{~m}$. Now, if $N_{1 H}=N_{1 W}=8, I R S_{2}$ has an approximate dimension of $4 m \times 4 m$.

4) Number of elements in $I R S_{1}\left(N_{1}\right)$ : The number of elements in $I R S_{1}$ determines the length $L$ of the array and its beam-width $\theta_{B W}$, where these parameter decide the spacing between two IRSs and the inter-element spacing in $I R S_{2}$. A larger value of $N_{1}$ with accurate phase shifting and a larger value of $d_{2}$ direct the beam to the desired direction, hence reducing the potential beam misalignment problem of our beam index modulation scheme.

5) Number of elements in $\operatorname{IRS}_{2}\left(N_{2}\right)$ : This determines the data rate of the system. A large value of $N_{2}$ gives a higher data rate. However, this will make the size of $I R S_{2}$ large. Hence, $N_{2}$ is restricted by the maximum affordable array dimension. 


\section{DETECTOR}

The detector has to recover the bits embedded both into the QAM/PSK symbol and the TA activation pattern in $I R S_{2}$. Explicitly, it has to detect $s$ and $\mathbf{b}$ from $\mathbf{y}$ in (2). Let $\mathbf{x}=\mathbf{b} s$ and $\mathbf{A}=\mathbf{H} \Theta$. Now, (2) can be written as:

$$
\mathbf{y}=\mathbf{A x}+\mathbf{w} .
$$

It is assumed that $\mathbf{A}$ is known at the receiver.

\section{A. Optimal Detector}

We first derive the optimal ML detector for the single symbol cases, i.e. for Scheme 1 and Scheme 3. Then we extent it to the multi symbol case of Scheme 2.

1) Single-Symbol Schemes: Consider the vector $\mathbf{x}$ in (11). Ideally in single-symbol schemes only one of the entries in $\mathbf{x}$ should be a non-zero value, since only one element receives the symbol. However, this will not be the case in practice, since the beamformer will introduce a non-zero power also in directions other than the required one. Hence, practically more than one element of $I R S_{2}$ receives the symbol. However, the power in the undesired beam-indices is much lower than that in the intended index and these powers depend on the beampattern of $I R S_{1}$. The data-dependent beam-index changes can be represented approximately by a beampattern rotation. Hence, (11) can be written as:

$$
\mathbf{y}=\mathbf{A} \Pi_{\mathbf{p}}+\mathbf{w},
$$

where $\mathbf{p}$ represents the vector of powers in the various indices of $\mathbf{x}$ and $\Pi_{\mathbf{p}}$ represents a particular permutation of the power pattern. Therefore, in order to identify the beam-index, we have to identify the power pattern permutation $\Pi_{\mathbf{p}}$. Now, $\mathbf{y} \sim \mathcal{C N}\left(\mathbf{A} \Pi_{\mathbf{p}}, \Sigma\right)$. Hence, the ML detector of this problem is formulated as:

$$
\begin{aligned}
& \min _{\Pi_{\mathbf{p}}}\left(\mathbf{y}-\mathbf{A} \Pi_{\mathbf{p}}\right)^{H} \Sigma^{-1}\left(\mathbf{y}-\mathbf{A} \Pi_{\mathbf{p}}\right) \\
\Longrightarrow & \max _{\Pi_{\mathbf{p}}} \mathcal{R} e\left\{\left(\mathbf{y}-\frac{1}{2} \mathbf{A} \Pi_{\mathbf{p}}\right)^{H} \Sigma^{-1} \mathbf{A} \Pi_{\mathbf{p}}\right\} .
\end{aligned}
$$

In general, the search problem (13) is NP-hard. However, in our case, there are only $N_{2} M$ different patterns corresponding to $N_{2}$ different beam indices and $M$ QAM/PSK symbols. Hence, a moderate-complexity search will give the optimal solution to (13).

2) Multi-Symbol Scheme: The ML detector (13) is also suitable for multi-symbol case. However, in this case, since there are $N_{T}$ desired beam-indices at a time, which interact with each other and thereby produce a large number of possible combinations $\Pi_{\mathbf{p}}$. Explicitly, $\left(\begin{array}{l}N_{2} \\ N_{T}\end{array}\right) M$ different patterns hypothesis must be tested for $N_{T}$ RSs in $I R S_{1}$. Hence the ML detector may no longer be a computationally attractable solution. Hence, in Section IV-B, we will be proposing a suboptimal compressed sensing (CS) aided detector, which can be used for any of the proposed schemes at a lower computational complexity.

\section{B. Suboptimal Compressed Sensing Detector}

The transmitted vector $\mathbf{x}$ in (11) is sparse, when the number of active elements (i.e., elements that receive the symbol) is much less than the total number of elements in $I R S_{2}$. Therefore, one can use an efficient sparse reconstruction algorithm [60], [61] for identifying the non-zero components in $\mathbf{x}$, which can be used to estimate $\mathbf{b}$. However, it should be noted that for the successful recovery of the sparse vector $\mathbf{x}$, there should be a sufficient number of measurements. This can be either achieved by having a sufficient number of RAs ( $N_{R}$ should be sufficiently large) or taking multiple measurements, which would naturally reduce the data rate. Finally, $s$ is obtained from the estimated $\mathbf{b}$ as:

$$
\hat{s}=\min _{s \in \mathcal{M}}\|\mathbf{y}-\mathbf{b} s\|^{2},
$$

where $\mathcal{M}$ is the constellation used.

\section{Complexity}

The optimal ML detector has to compute (13) for all possible combinations, which requires approximately on the order of $\left(N_{R}^{3}+N_{R} N_{2}\right)$ multiplications. This has to be done for each possible symbol. For the multi-symbol case, there are $\left(\begin{array}{l}N_{2} \\ N_{T}\end{array}\right) M$ possible symbols. Hence, the total computational complexity is approximately on the order of $\left(\begin{array}{c}N_{2} \\ N_{T}\end{array}\right) M\left(N_{R}^{3}+N_{R} N_{2}\right)$, which reduces to the order of $N_{2} M\left(N_{R}^{3}+N_{R} N_{2}\right)$ for single-symbol cases. On the other hand, if a greedy compressed sensing based suboptimal algorithm is used, the complexity will be reduced to the order of $N_{2} N_{R} N_{T}$, which is much lower than that of the optimal ML detector.

\section{Average Bit Error Rate Analysis}

In this section, we will estimate an upper bound for the average bit error rate (BER) of the optimal $\mathrm{ML}$ detector of Section IV-A. Let $\operatorname{Pr}\left\{\Pi_{\mathbf{p}}^{i} \rightarrow \Pi_{\mathbf{p}}^{j}\right\}$ represent the probability that the pattern $\Pi_{\mathbf{p}}^{i}$ is identified as $\Pi_{\mathbf{p}}^{j}$ and $v_{i, j}$ represent the number of bits in error between the two permutations $\Pi_{\mathbf{p}}^{i}$ and $\Pi_{\mathbf{p}}^{j}$. Then the average BER is formulated as:

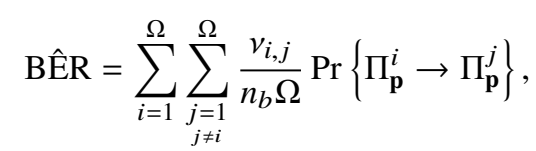

where $n_{b}$ is the total number of bits per channel use and $\Omega$ is the total number of possible permutations. Equation (15) assumes that all permutations are equally likely. The probability of symbol error $\operatorname{Pr}\left\{\Pi_{\mathbf{p}}^{i} \rightarrow \Pi_{\mathbf{p}}^{j}\right\}$ 
in (15) can be found as follows. When $\Pi_{\mathbf{p}}^{i}$ is transmitted, the detector identifies $\Pi_{\mathbf{p}}^{j}$ as the transmitted symbol based on:

$$
\underset{k}{\arg \max } \mathcal{R} e\left\{\left(\mathbf{y}-\frac{1}{2} \mathbf{A} \Pi_{\mathbf{p}}^{k}\right)^{H} \Sigma^{-1} \mathbf{A} \Pi_{\mathbf{p}}^{k}\right\}=j,
$$

where $\mathbf{y}$ is given in (12) in conjunction with $\Pi_{\mathbf{p}}=$ $\Pi_{\mathbf{p}}^{i}$. Let us define $r_{k}=\mathcal{R} e\left\{\left(\mathbf{y}-\frac{1}{2} \mathbf{A} \Pi_{\mathbf{p}}^{k}\right)^{H} \Sigma^{-1} \mathbf{A} \Pi_{\mathbf{p}}^{k}\right\}$. Hence, we have

$$
\operatorname{Pr}\left\{\Pi_{\mathbf{p}}^{i} \rightarrow \Pi_{\mathbf{p}}^{j}\right\}=\operatorname{Pr}\left\{\bigcap_{k \neq j} r_{j}>r_{k}\right\} .
$$

The computation of the probability of intersection of the event in (17) is very difficult. Hence, it is bounded using Fretchet's inequality [62] as follows:

$$
\operatorname{Pr}\left\{\bigcap_{k \neq j} r_{j}>r_{k}\right\} \leq \min _{k} \operatorname{Pr}\left\{r_{j}>r_{k}\right\} .
$$

In order to estimate the bound, the probabilities of $\operatorname{Pr}\left\{r_{j}>r_{k}\right\}$ have to be calculated for each $k \neq j$. Theorem 1 stated below gives an expression of the probability $\operatorname{Pr}\left\{r_{j}>r_{k}\right\}$.

Theorem 1. Let us assume the Rician channel model with $\mathbb{E}\{\mathbf{H}\}=\overline{\mathbf{H}}$ and that the row vectors of $\mathbf{H}$ are independent and identically distributed with covariance matrix $\tilde{\Sigma}_{c}$ and also let $\sigma_{2}^{2} \ll \sigma_{R}^{2}$. When $\Pi_{\mathbf{p}}^{i}$ is the actual signal transmitted and $\Theta$ is a constant matrix independent of $\mathbf{H}$, the probability of the events $r_{j}>r_{k}$, i.e. $\operatorname{Pr}\left\{r_{j}>r_{k}\right\}$ can be approximated as:

1) For $k=i$ :

$$
\operatorname{Pr}\left\{r_{j}>r_{i}\right\}=\frac{1}{2}\left[1-\sqrt{\frac{\beta_{1}}{1+\beta_{1}}} \sum_{n=0}^{N_{R}-1}\left(\begin{array}{c}
2 n \\
n
\end{array}\right)\left(\frac{1}{4\left(1+\beta_{1}\right)}\right)^{n}\right],
$$

where $\beta_{1}=\frac{\left(\Pi_{\mathbf{p}}^{i}-\Pi_{\mathbf{p}}^{j}\right)^{H} \Theta^{H}\left(\tilde{\Sigma}_{c}+\frac{1}{N_{R}} \overline{\mathbf{H}}^{H} \overline{\mathbf{H}}\right) \Theta\left(\Pi_{\mathbf{p}}^{i}-\Pi_{\mathbf{p}}^{j}\right)}{4 \sigma_{R}^{2}}$.

2) For $k \neq i$ and when $q_{R}=\mathcal{R} e\{q\} \neq 0$, where $q$ is defined in (48):

$\operatorname{Pr}\left\{r_{j}>r_{k}\right\}=\frac{1}{2}\left[1-\frac{1}{2} \sqrt{\frac{\beta_{2}}{1+\beta_{2}}} \sum_{n=0}^{N_{R}-1}\left(\begin{array}{c}2 n \\ n\end{array}\right)\left(\frac{1}{4\left(1+\beta_{2}\right)}\right)^{n}\right]$,

where $\beta_{2}=\frac{q_{R}^{2}}{\left(2+\sigma_{\kappa}^{2}\right) \sigma_{z_{1}}^{2}}$ and the constants $\sigma_{z_{1}}^{2}$ and $\sigma_{\kappa}^{2}$ are defined in (46) and (54), respectively.

3) For $k \neq i$ and when $q_{R}=0, \operatorname{Pr}\left\{r_{j}>r_{k}\right\}=\frac{1}{2}$.

Proof. See Appendix B for proof.

Finally, for each transmitted symbol $\Pi_{\mathbf{p}}^{i}$, the minimum value of $\operatorname{Pr}\left\{r_{j}>r_{k}\right\}, \forall k \neq j$ is computed using Theorem 1 and it is substituted for $\operatorname{Pr}\left\{\Pi_{\mathbf{p}}^{i} \rightarrow \Pi_{\mathbf{p}}^{j}\right\}$ into (15) for achieving the bound of the average BER. Note that (19) and (20) also hold for the Rayleigh channel, in which case the matrix $\left(\tilde{\Sigma}_{c}+\frac{1}{N_{R}} \overline{\mathbf{H}}^{H} \overline{\mathbf{H}}\right)$ is replaced by $\mathbf{I}$ to calculate $\beta_{1}$ and $\beta_{2}$.
The bounds derived for the average BER can be used for both Scheme 1 and Scheme 2. In the case of Scheme $1 \Omega=N_{2} M$ and $n_{b}=\log _{2} M+\left\lfloor\log _{2} N_{2}\right\rfloor$, whereas for Scheme 2, the corresponding values are $\Omega=\left(\begin{array}{l}N_{2} \\ N_{T}\end{array}\right) M$ and $n_{b}=\log _{2} M+\left\lfloor\log _{2}\left(\begin{array}{c}N_{2} \\ N_{T}\end{array}\right)\right\rfloor$. For Scheme $3, \Theta$ is no longer independent of $\mathbf{H}$ and therefore the bounds in Theorem 1 do not hold. However, the conditional probabilities derived in Appendix $\mathrm{C}$ can be used for Scheme 3 also. Based on this the unconditional probabilities can be derived using sampling method for computing the bound.

\section{Simulation Results}

Extensive simulations have been carried out to establish the performance of the proposed scheme on the system parameters. Explicitly, we studied the average BER of the proposed schemes vs. the SNR, the number of elements $\left(N_{2}\right)$ in $I R S_{2}$, the number of receivers $\left(N_{R}\right)$, the number of RSs in $I R S_{1}$ and the Rician factor, denoted by $K$. We have considered both the optimal ML detector and the low complexity compressed sensing detector in our performance evaluation. The system parameters used are given below.

- $I R S_{1}$ : A $100 \times 100$ rectangular array with spacing $2.5 \mathrm{~mm}$. This corresponds to half wavelength of the frequency.

- Distance between IRSs (D): $30 \mathrm{~m}$. This distance satisfies the far-field condition for $I R S_{1}$.

- IRS 2 : In general, an $8 \times 8$ rectangular array is used with inter element spacing of $d_{2}=60 \mathrm{~cm}$. However, these parameters are changed for the various performance studies, which is mentioned in the corresponding discussions.

We used 16 level $Q A M$ at $60 \mathrm{GHz}$ in all simulations. The results are shown in Fig. 6-9 for $10^{5}$ Monte Carlo runs. We used both Rician and Rayleigh fading channels. Throughout the simulations, it is assumed that the channel is perfectly known at the receiver. Channel estimation in IRS-aided systems is quite a challenge, since the IRS is passive and has no signal processing capability. However, the schemes adopted in [51], [63] could be used as a solution to the channel estimation problem.

In Fig. 6-Fig. 10, the proposed schemes 1 and 2 are referred as $S 1$ and $S 2$, respectively. $S 3$ represents the solution of (4), whereas $S 301$ and $S 302$ represent the solution of (7) and (10), respectively. $S 1$-Err represents the results of the proposed scheme 1 in the presence of channel estimation errors. ML and CS represent the results of optimal ML and compressed sensing based detectors, respectively and UB, the theoretical upper bound given in (18).

Fig. 5 shows the performance of scheme $S 1$ at various SNRs for different values of the Rician factor $(K)$. Observe from Fig. 5 that the performance of the low-complexity CS detector is inferior to that of the ML detector. As for the ML detector, the average 


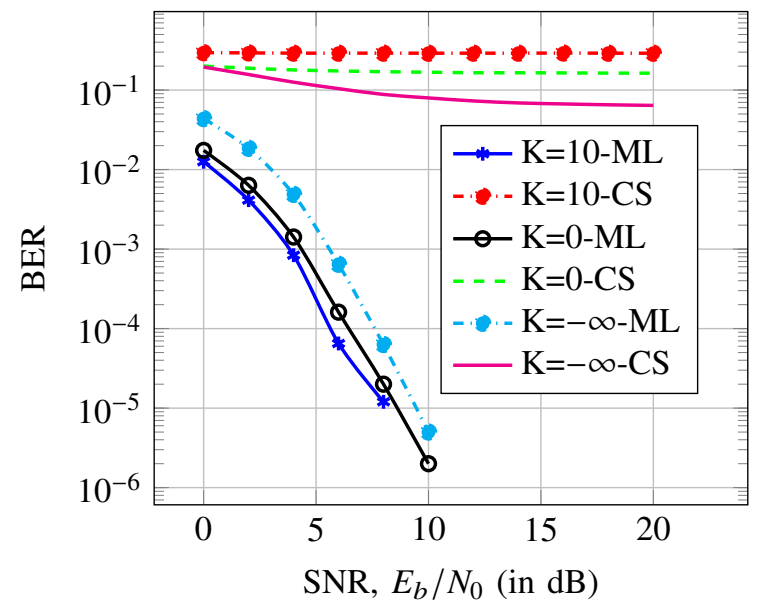

Fig. 5. S1: Comparison of ML and CS detectors by simulations. $K$ is the Rician factor in $d B$

BER tends to zero above $10 d B$ SNR, whereas it exhibits an error floor near 0.1 for the CS detector. The performance of the CS detector is improved, when the Rician factor $K$ decreases. This is because as $K$ decreases, the projection matrix $\mathbf{A}$ of the signal recovery becomes more random and the restricted isometric property [64] is improved and it is best for the Rayleigh channel. However, the performance of the ML detector is improved with $K$, since the LOS component is increased with $K$. This is also reflected by the upper bound seen in Fig. 6 .

Fig. 6 shows the performance of single-symbol beam-index modulation $(S 1)$ against the Rician factor $(K)$ for different SNRs. The average BER obtained through simulations is compared against the upper bound derived in Section V. The BER improvement vs. $K$ is due to increasing the LOS component. However, the variation in BER is only moderate, because the SNR is kept constant upon increasing $K$. Furthermore, the optimal ML detector has perfect channel knowledge. However, as $K$ increases, the gap between the simulation results and the corresponding upper bound is reduced. This is also observed at high SNRs. Therefore, it can be concluded that for both these cases, our bound becomes tighter.

The performance of the CS detector can be improved by increasing the number of RAs as shown in Fig. 7, where the average BER is plotted against $N_{R}$. This is plotted for Rayleigh channel, which gives the best performance for CS detector. The curves are shown for different number of elements in $I R S_{2}$ (i.e. $N_{2}$ ). Observe that for the same number of RAs, the performance degrades, as $N_{2}$ increases. However, as $N_{2}$ increases, the data rate will increase.

Fig. 8 compares the ML detector's performance for the three proposed schemes in terms of their average BER for Rician factor $K=0 d B$. For $S 2$, we used $N_{T}=2$, i.e. the number of RSs in $I R S_{1}$ is two. This is because, if $N_{T}$ is large, the complexity of optimal ML decoding will escalate. For fair comparison, the

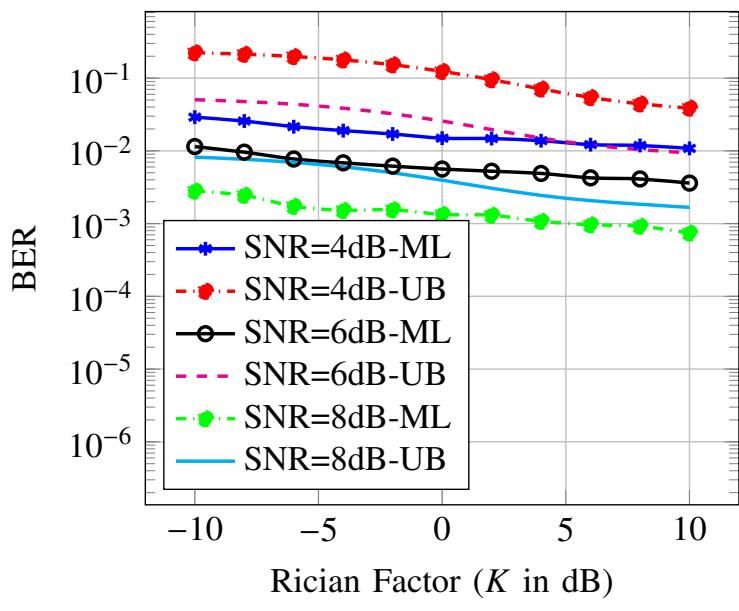

Fig. 6. $S 1$ : Comparison of the average BER and the theoretical upper bound (UB).

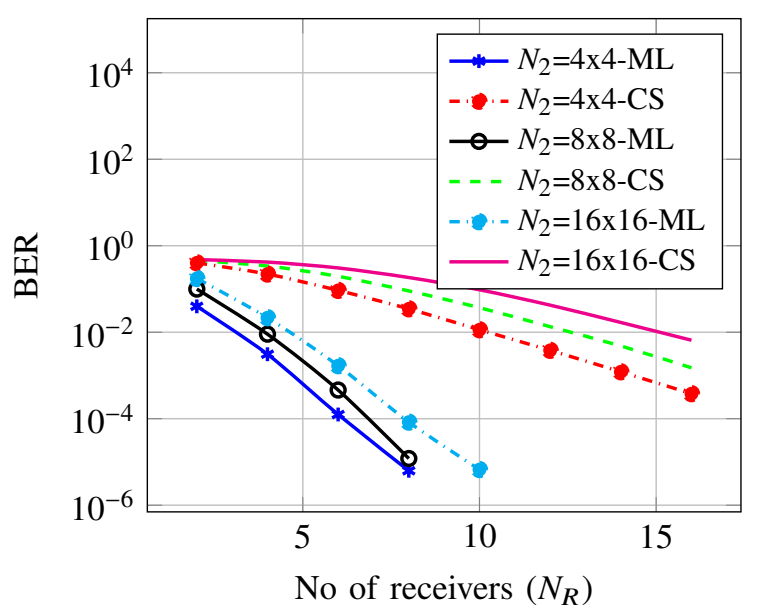

Fig. 7. $S 1$ : Effect of the number of receivers $\left(N_{R}\right)$ on the BER.

number of elements in all three cases are kept the same. Therefore, for $S 3$, where the optimization is to be carried out in an array, a $2 \times 2$ element array is considered to form a single RS. Hence, the effective dimension of $I R S_{2}$ in $S 3$ is $4 \times 4$, while it is $8 \times 8$ in the case of $S 1$ and $S 2$. Hence, the data rate will be lowest for $S 3$, whereas it is the highest for $S 2$, since there are more RSs in $I R S_{1}$. The data rate for $S 1, S 2$ and $S 3$ are 10, 14 and 8, respectively. In Fig. 8, the legends $S 3 O 1$ and $S 3 O 2$ represent the results of two optimization methods, i.e. the solution of (7) and that of (10), respectively. Both these schemes perform better than $S 1$ and $S 2$. This is because there is an increase in the received SNR due to optimization. In addition in S3, the modulating symbol is embedded in 4 elements, which gives an additional performance improvement. This makes the BER gap between the curves of $S 3$ and the other schemes substantial. Observe that $S 3 O 1$ performs marginally better than $S 3 O 2$. This is because $S 302$ maximizes the lower bound, whereas $S 301$ operates on the exact function. Note that $S 301$ performs almost similar to the solution of 


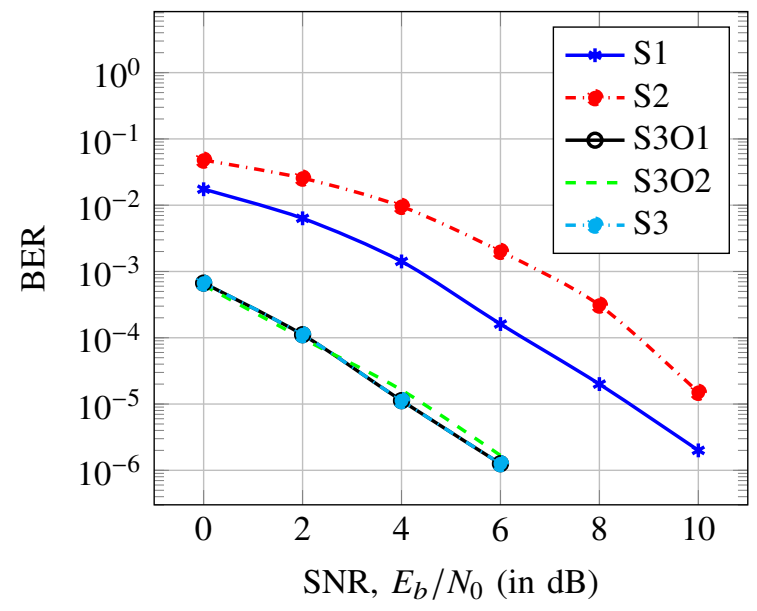

Fig. 8. Comparison of three different schemes.

the exact equation (4). The performance of $S 2$ is approximately $2 d B$ worse than that of $S 1$. There are two differences between these two schemes. In $S 2$, the same QAM/PSK symbol is carried by more than one elements in $I R S_{2}$. Hence, the probability of error in decoding the modulating symbol is reduced compared to $S 1$. However, the information carried by the beamindex is higher in the case of $S 2$, whose probability of decoding error will be higher than that of $S 1$. The average BER reflects these two opposite effects.

Fig. 9 shows the effect of the number of RSs $\left(N_{T}\right)$ in $I R S_{1}$ on the BER performance in $S 3$ for different number of receivers $\left(N_{R}\right)$ for CS detector. Similar to Fig. 7, this is also under Rayleigh channel condition. The average BER increases as $N_{T}$ increases, which can be reduced by increasing the number of receivers. However, as $N_{T}$ increases, the data rate increases. In this case, for the single RS case (which is equivalent to $S 1$ ), the data rate is $10 \mathrm{bpcu}$, while it is $14,23,30$ and 36 for $N_{T}=2,4,6 \& 8$, respectively. Finally, in Fig. 10, the effect of channel estimation errors is demonstrated. The true channel coefficients are corrupted by adding noise having a variance of $\sigma_{R}^{2}$, which affects both the optimization as well as detection. The average BER is shown in the figure both with and without channel estimation. It can be seen that both $S 1$ and $S 2$ have approximately $2-3 d B$ performance degradation owing to the channel estimation error, whereas this gap is in excess of $4 d B$ for $S 3$. This is because, in $S 3$, the contaminated channel information is used both for optimization and detection.

\section{CONCLUSIONS}

We proposed beam index modulation for millimeter wave communication exploiting the benefits of IRSs. The proposed scheme has three main advantages: 1) It achieves low-cost beamforming by using IRS for applying phase shifts, 2) it is capable of achieving reliable communication with the help of multiple IRSs in non-LOS scenarios, and 3) it sends additional in-

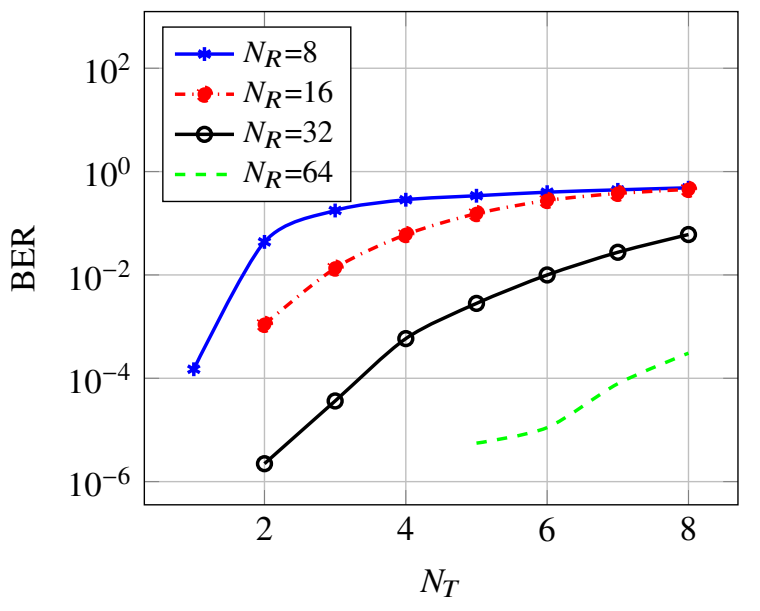

Fig. 9. S2: Effect of the number of reflecting surfaces in $I R S_{1}$ $\left(N_{T}\right)$

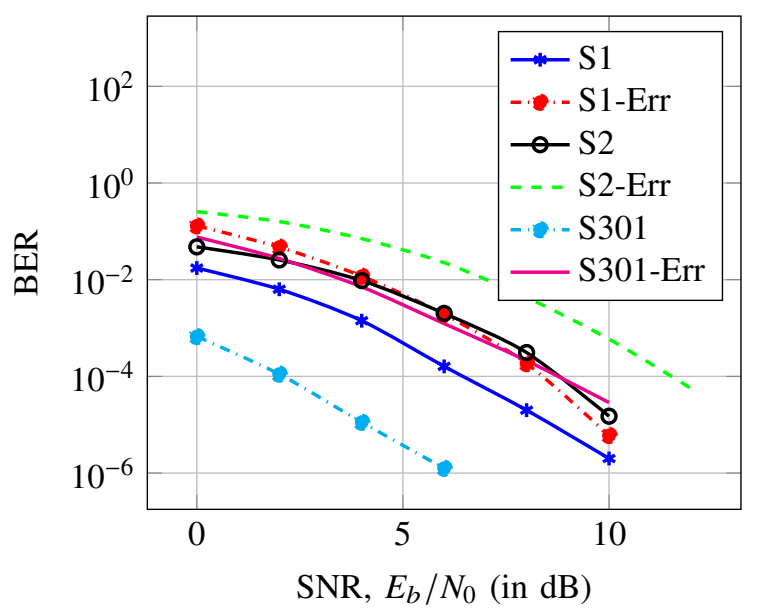

Fig. 10. Effect of channel estimation error.

formation using beam-index modulation without any additional cost. Furthermore, we developed the optimal ML detector and a low-complexity compressed sensing detector for the proposed schemes. An upper bound of the average BER of the optimal ML detector is also achieved. Finally, the performance of the proposed schemes was evaluated through extensive simulations.

\section{APPENDIX A PROOF OF LEMMA 1}

$$
\begin{gathered}
\left\|\mathbf{H}_{Q} \Theta_{Q} \mathbf{b}_{Q} s\right\|^{2}=\left(\mathbf{b}_{Q} s\right)^{H} \Theta_{Q}^{H} \mathbf{H}_{Q}^{H} \mathbf{H}_{Q} \Theta_{Q}\left(\mathbf{b}_{Q} s\right) \\
=\operatorname{Tr}\left\{\left(\mathbf{b}_{Q} s\right)^{H} \Theta_{Q}^{H} \mathbf{H}_{Q}^{H} \mathbf{H}_{Q} \Theta_{Q}\left(\mathbf{b}_{Q} s\right)\right\} \\
=\operatorname{Tr}\left\{\Theta_{Q}^{H} \mathbf{H}_{Q}^{H} \mathbf{H}_{Q} \Theta_{Q}\left(\mathbf{b}_{Q} s\right)\left(\mathbf{b}_{Q} s\right)^{H}\right\} .
\end{gathered}
$$

Note that $\Theta_{Q}^{H} \mathbf{H}_{Q}^{H} \mathbf{H}_{Q} \Theta_{Q}$ and $\left(\mathbf{b}_{Q} s\right)\left(\mathbf{b}_{Q} s\right)^{H}$ are positive definite matrices. Therefore applying [65, Theorem 2] on (21) results in (8). Now, since $H_{Q}$ is a random matrix and if $R \geq Q, \mathbf{H}_{Q}^{H} \mathbf{H}_{Q}$ will be a full-rank matrix with probability 1 and consequently $\lambda_{\min }\left(\Theta_{Q}^{H} \mathbf{H}_{Q}^{H} \mathbf{H}_{Q} \Theta_{Q}\right)>0$. 
APPENDIX B

PROOF OF THEOREM 1

Lemma 2 of Appendix $\mathrm{C}$ gives the conditional probability $\operatorname{Pr}\left\{r_{j}>r_{k} \mid \mathbf{A}\right\}$. Explicitly, the conditional probabilities are in the form of complementary error function ( $Q$-functions). The distributions of the arguments of these $Q$-functions are derived in Lemma 3 in Appendix D. Therefore, the unconditional probabilities can be calculated by taking expectation of conditional probabilities (36) with respect to the corresponding distributions of their arguments in (43) and (44).

For $k=i$, the argument of the conditional probability is a $\Gamma$-distributed random variable with rate parameter $b=\frac{2 \sigma_{R}^{2}}{\left(\Pi_{\mathbf{p}}^{i}-\Pi_{\mathbf{p}}^{j}\right)^{H} \Theta^{H} \Sigma_{c} \Theta\left(\Pi_{\mathbf{p}}^{i}-\Pi_{\mathbf{p}}^{j}\right)}$ and shape parameter $k=N_{R}$, i.e., $f_{\gamma_{i j}}\left(\gamma_{i j}\right)=\frac{1}{\Gamma(k)} b^{k} \gamma_{i j}^{k-1} e^{-b \gamma_{i j}}$. The unconditional probability in this case is

$$
\operatorname{Pr}\left\{r_{j}>r_{i}\right\}=\int_{0}^{\infty} Q\left(\sqrt{\gamma_{i j}}\right) f_{\gamma_{i j}}\left(\gamma_{i j}\right) d \gamma_{i j}
$$

The closed-form expression for (22) given in [66, Eq. (A12)] can be applied to get (19). This proves the first part of the theorem.

For $k \neq i$, the probability is computed as follows. First we will consider the case of $q_{R} \neq 0$. By exploiting the relationship $Q(x)=1-Q(-x)$, the probability $\operatorname{Pr}\left\{r_{j}>r_{k}\right\}$ can be written as:

$$
\begin{aligned}
\operatorname{Pr} & \left\{r_{j}>r_{k}\right\}=\int_{-\infty}^{\infty} Q(\kappa) f_{\kappa}(\kappa) d \kappa \\
& =\int_{-\infty}^{0}(1-Q(-\kappa)) f_{\kappa}(\kappa) d \kappa+\int_{0}^{\infty} Q(\kappa) f_{\kappa}(\kappa) d \kappa \\
& =\int_{0}^{\infty} f_{\kappa}(-\kappa) d \kappa+\int_{0}^{\infty} Q(\kappa)\left(f_{\kappa}(\kappa)-f_{\kappa}(-\kappa)\right) d \kappa \\
& =\tilde{C}\left(I_{1}+I_{2}\right),
\end{aligned}
$$

where $\tilde{C}=\frac{2 \Gamma\left(2 N_{R}\right)}{\Gamma\left(N_{R}\right) \sqrt{\pi \sigma_{K}^{2}}}\left(\frac{v-1}{2 v}\right)^{N_{R}}$ is a constant term and

$$
I_{1}=\int_{0}^{\infty} e^{-\frac{2 v-1}{2 v \sigma_{\kappa}^{2}} \kappa^{2}} D_{-2 N_{R}}\left(\sqrt{\frac{2}{v \sigma_{\kappa}^{2}}} \kappa\right) d \kappa,
$$

while

$$
\begin{aligned}
I_{2}=\int_{0}^{\infty} & Q(\kappa) e^{-\frac{2 v-1}{2 v \sigma_{\kappa}^{2}} \kappa^{2}}\left[D_{-2 N_{R}}\left(-\sqrt{\frac{2}{v \sigma_{\kappa}^{2}}} \kappa\right)\right. \\
& \left.-D_{-2 N_{R}}\left(\sqrt{\frac{2}{v \sigma_{\kappa}^{2}}} \kappa\right)\right] d \kappa .
\end{aligned}
$$

Note $D_{(.)}($.$) is the parabolic cylinder function [67, pp.$ 45] and it can be written in terms Kummer's confluent hypergeometric function ${ }_{1} F_{1}($.$) as [68$, pp. $39(23)]$

$$
\begin{aligned}
D_{K}(z) & =2^{\frac{K}{2}} \sqrt{\pi} e^{-\frac{z^{2}}{4}}\left[\frac{1}{\Gamma\left(\frac{1-K}{2}\right)}{ }_{1} F_{1}\left(-\frac{K}{2} ; \frac{1}{2} ; \frac{z^{2}}{2}\right)\right. \\
& \left.-\frac{z}{\sqrt{2} \Gamma\left(-\frac{K}{2}\right)}{ }_{1} F_{1}\left(\frac{1-K}{2} ; \frac{3}{2} ; \frac{z^{2}}{2}\right)\right] .
\end{aligned}
$$

Let us substitute $\kappa=+\sqrt{t}$ into (24) and expand $D_{-2 N_{R}(\text {.) }}$ using (26). Note that $d \kappa=\frac{1}{2 \sqrt{t}} d t$. Therefore $I_{1}$ becomes:

$$
\begin{aligned}
I_{1} & =\frac{\sqrt{\pi} 2^{-\left(N_{R}+1\right)}}{\Gamma\left(N_{R}+\frac{1}{2}\right)} \int_{0}^{\infty} t^{-\frac{1}{2}} e^{-\frac{t}{\sigma_{\kappa}^{2}}}{ }_{1} F_{1}\left(N_{R} ; \frac{1}{2} ; \frac{t}{v \sigma_{\kappa}^{2}}\right) d t \\
& -\frac{2^{-\left(N_{R}+1\right)}}{\Gamma\left(N_{R}\right)} \sqrt{\frac{\pi}{v \sigma_{\kappa}^{2}}} \int_{0}^{\infty} e^{-\frac{t}{\sigma_{K}^{2}}}{ }_{1} F_{1}\left(N_{R}+\frac{1}{2} ; \frac{3}{2} ; \frac{t}{v \sigma_{\kappa}^{2}}\right) d t .
\end{aligned}
$$

Now, the difference in (25) is formulated as:

$$
\begin{aligned}
\Delta & =D_{-2 N_{R}}\left(-\sqrt{\frac{2 t}{v \sigma_{\kappa}^{2}}}\right)-D_{-2 N_{R}}\left(\sqrt{\frac{2 t}{v \sigma_{\kappa}^{2}}}\right) \\
& =\frac{2 e^{-\frac{t}{2 v \sigma_{\kappa}^{2}}} 2^{-N_{R}}}{\Gamma\left(N_{R}\right)} \sqrt{\frac{\pi t}{v \sigma_{\kappa}^{2}}}{ }_{1} F_{1}\left(N_{R}+\frac{1}{2} ; \frac{3}{2} ; \frac{t}{v \sigma_{\kappa}^{2}}\right) .
\end{aligned}
$$

In order to evaluate $I_{2}$, first we express the $Q$-function in terms of the complimentary error function as $Q(x)=$ $\frac{1}{2} \operatorname{erfc}\left(\frac{x}{\sqrt{2}}\right)$ [69, pp. 40], and then subsequently it is expressed in terms of the hypergeometric function as [70]:

$$
Q(x)=\frac{1}{2}-\frac{x}{\sqrt{2 \pi}}{ }_{1} F_{1}\left(\frac{1}{2} ; \frac{3}{2} ;-\frac{x^{2}}{2}\right) .
$$

Upon substituting (28) and (29) into (25), $I_{2}$ becomes:

$$
\begin{gathered}
I_{2}=\frac{2^{-\left(N_{R}+1\right)}}{\Gamma\left(N_{R}\right)} \sqrt{\frac{\pi}{v \sigma_{\kappa}^{2}}} \int_{0}^{\infty} e^{-\frac{t}{\sigma_{\kappa}^{2}}}{ }_{1} F_{1}\left(N_{R}+\frac{1}{2} ; \frac{3}{2} ; \frac{t}{v \sigma_{\kappa}^{2}}\right) d t \\
-\frac{2^{-N_{R}}}{\Gamma\left(N_{R}\right)} \sqrt{\frac{1}{2 v \sigma_{\kappa}^{2}}} \int_{0}^{\infty} t^{\frac{1}{2}} e^{-\frac{t}{\sigma_{\kappa}^{2}}}{ }_{1} F_{1}\left(\frac{1}{2} ; \frac{3}{2} ;-\frac{t}{2}\right) \\
{ }_{1} F_{1}\left(N_{R}+\frac{1}{2} ; \frac{3}{2} ; \frac{t}{v \sigma_{\kappa}^{2}}\right) d t .
\end{gathered}
$$

Note that the second term of the RHS in (27) and the first term of RHS in (30) will get cancelled. Hence $\operatorname{Pr}\left\{r_{j}>r_{k}\right\}$ will become:

$$
\begin{aligned}
\operatorname{Pr} & \left\{r_{j}>r_{k}\right\}=C_{1} \int_{0}^{\infty} t^{-\frac{1}{2}} e^{-\frac{t}{\sigma_{\kappa}^{2}}}{ }_{1} F_{1}\left(N_{R} ; \frac{1}{2} ; \frac{t}{v \sigma_{\kappa}^{2}}\right) d t \\
& -C_{2} \int_{0}^{\infty} t^{\frac{1}{2}} e^{-\frac{t}{\sigma_{\kappa}^{2}}}{ }_{1} F_{1}\left(\frac{1}{2} ; \frac{3}{2} ;-\frac{t}{2}\right){ }_{1} F_{1}\left(N_{R}+\frac{1}{2} ; \frac{3}{2} ; \frac{t}{v \sigma_{\kappa}^{2}}\right) d t \\
& =C_{1} I_{3}+C_{2} I_{4},
\end{aligned}
$$

where $C_{1}=\frac{1}{2 \sqrt{\pi \sigma_{\kappa}^{2}}}\left(\frac{v-1}{v}\right)^{N_{R}}$ and $C_{2}=$ $\frac{1}{\pi \sigma_{\kappa}^{2} \sqrt{2 v}} \frac{\Gamma\left(N_{R}+\frac{1}{2}\right)}{\Gamma\left(N_{R}\right)}\left(\frac{v-1}{v}\right)^{N_{R}}$. Note that we have exploited the relationship $\Gamma(x) \Gamma\left(x+\frac{1}{2}\right)=\frac{\sqrt{\pi}}{2^{2 x-1}} \Gamma(2 x)$ 
[71, Theorem 6] for reducing $C_{1}$ and $C_{2}$. Now, $I_{3}$ is expressed using equation [72, pp. 822, equation(7.621(4))] and it is given below:

$$
\begin{aligned}
I_{3} & =\int_{0}^{\infty} t^{-\frac{1}{2}} e^{-\frac{t}{\sigma_{\kappa}^{2}}}{ }_{1} F_{1}\left(N_{R} ; \frac{1}{2} ; \frac{t}{v \sigma_{\kappa}^{2}}\right) d t \\
& =\Gamma\left(\frac{1}{2}\right) \sqrt{\sigma_{\kappa}^{2}}{ }_{2} F_{1}\left(N_{R}, \frac{1}{2} ; \frac{1}{2} ; \frac{1}{v}\right) \\
& =\sqrt{\pi \sigma_{\kappa}^{2}}\left(1-\frac{1}{v}\right)^{-N_{R}},
\end{aligned}
$$

where ${ }_{2} F_{1}(a, b ; c ; z)$ is Gauss' Hypergeometric function [67, pp. 42]. Observe that ${ }_{2} F_{1}(a, b ; b ; z)={ }_{1} F_{0}(a ; z)=$ $(1-z)^{-a}[73, \mathrm{~A} 1]$. In order to evaluate the integral $I_{4}$, we make the substitution $z=\frac{t}{v \sigma_{\kappa}^{2}}$ and use [72, pp. 823, equation (7.622(1))] and the integral becomes:

$$
\begin{aligned}
I_{4} & =\int_{0}^{\infty} t^{\frac{1}{2}} e^{-\frac{t}{\sigma_{K}^{2}}}{ }_{1} F_{1}\left(\frac{1}{2} ; \frac{3}{2} ;-\frac{t}{2}\right){ }_{1} F_{1}\left(N_{R}+\frac{1}{2} ; \frac{3}{2} ; \frac{t}{v \sigma_{K}^{2}}\right) d t \\
& =\left(v \sigma_{K}^{2}\right)^{\frac{3}{2}} \int_{0}^{\infty} z^{\frac{1}{2}} e^{-v z}{ }_{1} F_{1}\left(\frac{1}{2} ; \frac{3}{2} ;-\frac{v \sigma_{K}^{2}}{2} z\right){ }_{1} F_{1}\left(N_{R}+\frac{1}{2} ; \frac{3}{2} ; z\right) d z \\
& =\sqrt{\frac{\pi\left(\sigma_{K}^{2}\right)^{3}}{2\left(2+\sigma_{K}^{2}\right)}}{ }_{2} F_{1}\left(N_{R}+\frac{1}{2}, \frac{1}{2} ; \frac{3}{2} ;-\frac{\sigma_{K}^{2}}{(v-1)\left(2+\sigma_{K}^{2}\right)}\right)\left(\frac{v-1}{v}\right)^{-\left(N_{R}+\frac{1}{2}\right)}
\end{aligned}
$$

Now we apply the transformations ${ }_{2} F_{1}(a, b ; c ; z)=(1-z)^{-b}{ }_{2} F_{1}\left(c-a, b ; c ; \frac{z}{z-1}\right)$ [74] and ${ }_{2} F_{1}(a, b ; b+1 ; z)=b z^{-b} \mathcal{B}_{z}(b, 1-a)$ [75], when (33) becomes:

$$
I_{4}=\frac{\sigma_{\kappa}^{2}}{2} \sqrt{\frac{\pi v}{2}}\left(\frac{v-1}{v}\right)^{-N_{R}} \mathcal{B} \frac{\sigma_{\kappa}^{2}}{2(v-1)+v \sigma_{\kappa}^{2}}\left(\frac{1}{2}, N_{R}\right),
$$

where $\mathcal{B}_{z}(a, b)=\mathcal{B}(a, b) z^{a} \sum_{k=0}^{b-1} \frac{(a)_{(k)}}{k !}(1-z)^{k}$ is the incomplete Beta function [76] with $\mathcal{B}(a, b)=\frac{\Gamma(a) \Gamma(b)}{\Gamma(a+b)}$ being the Beta function and $(a)_{(k)}$ is the Pochhammer symbol. Finally upon substituting (32) and (34) into (31), we arrive at:

$\operatorname{Pr}\left\{r_{j}>r_{k}\right\}=\frac{1}{2}$

$-\frac{1}{4} \sqrt{\frac{\sigma_{K}^{2}}{2(v-1)+v \sigma_{\kappa}^{2}}} \sum_{n=0}^{N_{R}-1} \frac{\left(\frac{1}{2}\right)(n)}{n !}\left(1-\frac{\sigma_{K}^{2}}{2(v-1)+v \sigma_{K}^{2}}\right)^{n}$

Now substituting for $v$ and the Pochhammer symbol $(a)_{(n)}=\frac{\Gamma(n+a)}{\Gamma(a)}$ [77] will give the second term in the RHS of (20).

Finally, when $q_{R}=0$, the distribution of $\kappa$ is zero mean Gaussian. Hence, in this case $I_{2}$ in (23) will be zero, while $I_{1}=\frac{1}{2}$ and the constant $\tilde{C}=1$, which completes the proof.

\section{APPENDIX C}

Conditional Probability $\operatorname{Pr}\left\{r_{j}>r_{k} \mid \mathbf{A}\right\}$

Lemma 2. Let $\Pi_{\mathbf{p}}^{i}$ be the transmitted signal. Then, we have

$$
\operatorname{Pr}\left\{r_{j}>r_{k} \mid \mathbf{A}\right\}=\left\{\begin{array}{ll}
Q\left(\sqrt{\gamma_{i j}}\right), & \text { if } k=i \\
Q(\kappa), & \text { otherwise }
\end{array},\right.
$$

where $Q(x)=\frac{1}{\sqrt{2 \pi}} \int_{x}^{\infty} \mathbf{e}^{-\frac{t^{2}}{2}} d x$ is the complementary error function and

$$
\gamma_{k j}=\frac{1}{2}\left(\Pi_{\mathbf{p}}^{k}-\Pi_{\mathbf{p}}^{j}\right)^{H} \mathbf{A}^{H} \Sigma^{-1} \mathbf{A}\left(\Pi_{\mathbf{p}}^{k}-\Pi_{\mathbf{p}}^{j}\right),
$$

and

$$
\kappa=\frac{\gamma_{i k}-\gamma_{i j}}{\sqrt{\gamma_{k j}}}
$$

Proof. First the conditional probability $\operatorname{Pr}\left\{r_{j}>r_{k} \mid \mathbf{A}\right\}$ is estimated for $k \neq i$ when $\Pi_{\mathbf{p}}^{i}$ is transmitted as follows. The event $r_{j}>r_{k}$ is

$\mathcal{R e}\left\{\left(\mathbf{y}-\frac{1}{2} \mathbf{A} \Pi_{\mathbf{p}}^{k}\right)^{H} \Sigma^{-1} \mathbf{A} \Pi_{\mathbf{p}}^{k}\right\}<\mathcal{R e}\left\{\left(\mathbf{y}-\frac{1}{2} \mathbf{A} \Pi_{\mathbf{p}}^{j}\right)^{H} \Sigma^{-1} \mathbf{A} \Pi_{\mathbf{p}}^{j}\right\}$.

Using (12), (39) can be written as:

$$
\begin{aligned}
\mathcal{R e} & \left\{\left(\mathbf{w}+\mathbf{A} \Pi_{\mathbf{p}}^{i}\right)^{H} \Sigma^{-1} \mathbf{A}\left(\Pi_{\mathbf{p}}^{k}-\Pi_{\mathbf{p}}^{j}\right)\right\} \\
& <\operatorname{Re}\left\{-\frac{1}{2}\left(\Pi_{\mathbf{p}}^{j}-\Pi_{\mathbf{p}}^{k}\right)^{H} \mathbf{A}^{H} \Sigma^{-1} \mathbf{A}\left(\Pi_{\mathbf{p}}^{j}+\Pi_{\mathbf{p}}^{k}\right)\right\} .
\end{aligned}
$$

Hence, $\operatorname{Pr}\left\{r_{j}>r_{k} \mid \mathbf{A}\right\}=\operatorname{Pr}\left\{\eta<g_{j k} \mid \mathbf{A}\right\}$, where $\eta=$ $\mathcal{R} e\left\{\left(\mathbf{w}+\mathbf{A} \Pi_{\mathbf{p}}^{i}\right)^{H} \Sigma^{-1} \mathbf{A}\left(\Pi_{\mathbf{p}}^{k}-\Pi_{\mathbf{p}}^{j}\right)\right\}$ and $g_{j k}$ is the RHS of (40). Finally, note that $\eta \sim \mathcal{N}\left(\mu_{j k}, \gamma_{j k}\right)$, where $\mu_{j k}=\mathcal{R} e\left\{\left(\Pi_{\mathbf{p}}^{i}\right)^{H} \mathbf{A}^{H} \Sigma^{-1} \mathbf{A}\left(\Pi_{\mathbf{p}}^{k}-\Pi_{\mathbf{p}}^{j}\right)\right\}$ and $\gamma_{j k}$ is defined in (37). Now, let us make a substitution $\tilde{\eta}=\frac{\eta-\mu_{j k}}{\sqrt{\gamma_{j k}}}$. Clearly, $\tilde{\eta}$ is a standard normal random variable and hence the probability in (37) can be written in the form of the $Q$-function as:

$$
\operatorname{Pr}\left\{r_{j}>r_{k} \mid \mathbf{A}\right\}=\operatorname{Pr}\left\{\tilde{\eta}<-\frac{s_{j k}}{\sqrt{\gamma_{j k}}}\right\}=Q\left(\frac{s_{j k}}{\sqrt{\gamma_{j k}}}\right),
$$

where we have

$$
\begin{aligned}
s_{j k} & =-\left(g_{j k}-\mu_{j k}\right) \\
& =\operatorname{Re}\left\{\frac{1}{2}\left(\Pi_{\mathbf{p}}^{j}-\Pi_{\mathbf{p}}^{k}\right)^{H} \mathbf{A}^{H} \Sigma^{-1} \mathbf{A}\left(\Pi_{\mathbf{p}}^{j}+\Pi_{\mathbf{p}}^{k}-2 \Pi_{\mathbf{p}}^{i}\right)\right\} \\
& =\operatorname{Re}\left\{\frac{1}{2}\left(\left(\Pi_{\mathbf{p}}^{j}-\Pi_{\mathbf{p}}^{i}\right)-\left(\Pi_{\mathbf{p}}^{k}-\Pi_{\mathbf{p}}^{i}\right)\right)^{H}\right. \\
& \left.\mathbf{A}^{H} \Sigma^{-1} \mathbf{A}\left(\left(\Pi_{\mathbf{p}}^{j}-\Pi_{\mathbf{p}}^{i}\right)+\left(\Pi_{\mathbf{p}}^{k}-\Pi_{\mathbf{p}}^{i}\right)\right)\right\} \\
& =\gamma_{i j}-\gamma_{i k} .
\end{aligned}
$$

When $k=i$, we have $\gamma_{i k}=0$ and therefore (41) becomes $\operatorname{Pr}\left\{r_{j}>r_{i} \mid \mathbf{A}\right\}=Q\left(\sqrt{\gamma_{i j}}\right)$. This completes the proof.

\section{APPENDIX D}

Distribution OF $\gamma_{m n}$ AND $\kappa$

Lemma 3. Under the assumptions of Theorem 1, 
1) The random variable $\gamma_{k j}$ defined in (37) is a $\Gamma$ distributed random variable, i.e.,

$$
\gamma_{k j} \sim \Gamma\left(N_{R}, \frac{2 \sigma_{R}^{2}}{\left(\Pi_{\mathbf{p}}^{k}-\Pi_{\mathbf{p}}^{j}\right)^{H} \Theta^{H} \Sigma_{c} \Theta\left(\Pi_{\mathbf{p}}^{k}-\Pi_{\mathbf{p}}^{j}\right)}\right) .
$$

2) When $q_{R} \neq 0$, the distribution of the random variable $\kappa$ defined in (38) is

$$
f_{\kappa}(\kappa)=\frac{2 \Gamma\left(2 N_{R}\right) e^{-\frac{2 v-1}{2 v \sigma_{\kappa}^{2}} \kappa^{2}}}{\Gamma\left(N_{R}\right) \sqrt{\pi \sigma_{\kappa}^{2}}}\left(\frac{v-1}{2 v}\right)^{N_{R}} D_{-2 N_{R}}\left(-\sqrt{\frac{2}{v \sigma_{\kappa}^{2}} \kappa}\right),
$$

where $D_{K}($.$) is the Parabolic cylinder function$ [67, pp. 45] and the parameters are defined in Theorem 1.

3) For $q_{R}=0, \kappa \sim \mathcal{N}\left(0, \frac{\sigma_{\kappa}^{2}}{2}\right)$, where $\sigma_{\kappa}^{2}$ is defined in (54)

Proof. When we have $\sigma_{2}^{2} \ll \sigma_{R}^{2}, \gamma_{k j}$ in (37) can be approximated as:

$$
\gamma_{k j} \approx \frac{1}{2 \sigma_{R}^{2}}\left(\Pi_{\mathbf{p}}^{k}-\Pi_{\mathbf{p}}^{j}\right)^{H} \mathbf{A}^{H} \mathbf{A}\left(\Pi_{\mathbf{p}}^{k}-\Pi_{\mathbf{p}}^{j}\right) .
$$

Recall that $\mathbf{A}=\mathbf{H} \Theta$. Under the assumptions of Theorem 1, $\mathbf{H}^{H} \mathbf{H}$ obeys a non-central complex Wishart distribution, i.e., $\mathbf{H}^{H} \mathbf{H} \sim \mathcal{W}\left(N_{R}, \overline{\mathbf{H}}, \tilde{\Sigma}_{c}\right)$. This can be approximated as a central complex Wishart distribution having the covariance matrix of $\Sigma_{c}=\tilde{\Sigma}_{c}+$ $\frac{1}{N_{R}} \overline{\mathbf{H}}^{H} \overline{\mathbf{H}}$ [78] ${ }^{1}$. If we use this approximation, it can be assumed that each entry of $\mathbf{H}$ is distributed according to $\mathcal{C N}\left(0, \Sigma_{c}\right)$. Hence, the random variable $\mathbf{z}=\frac{1}{\sqrt{2 \sigma_{R}^{2}}} \mathbf{A}\left(\Pi_{\mathbf{p}}^{i}-\Pi_{\mathbf{p}}^{j}\right)$ is distributed according to $C \mathcal{N}\left(0, \frac{1}{2 \sigma_{R}^{2}}\left(\Pi_{\mathbf{p}}^{i}-\Pi_{\mathbf{p}}^{j}\right)^{H} \Theta^{H} \Sigma_{c} \Theta\left(\Pi_{\mathbf{p}}^{i}-\Pi_{\mathbf{p}}^{j}\right) \mathbf{I}\right)$. Hence, $\gamma=\mathbf{z}^{H} \mathbf{z}$ is a Gamma distributed variable having the distribution function of (43). This proves the first part of the Lemma.

The rest of the Lemma is proved as follows. Define $\mathbf{z}_{1}=\frac{1}{\sqrt{2 \sigma_{R}^{2}}} \mathbf{A}\left(\left(\Pi_{\mathbf{p}}^{j}-\Pi_{\mathbf{p}}^{i}\right)-\left(\Pi_{\mathbf{p}}^{k}-\Pi_{\mathbf{p}}^{i}\right)\right)$ and $\mathbf{z}_{2}=$ $\frac{1}{\sqrt{2 \sigma_{R}^{2}}} \mathbf{A}\left(\left(\Pi_{\mathbf{p}}^{j}-\Pi_{\mathbf{p}}^{i}\right)+\left(\Pi_{\mathbf{p}}^{k}-\Pi_{\mathbf{p}}^{i}\right)\right)$. Similar to the case of first part of this Lemma, the distributions of $\mathbf{z}_{1}$ and $\mathbf{z}_{2}$ can be approximated as $\mathbf{z}_{1} \sim \mathcal{C N}\left(0, \sigma_{z_{1}}^{2} \mathbf{I}\right)$ and $\mathbf{z}_{2} \sim \mathcal{C N}\left(0, \sigma_{z_{2}}^{2} \mathbf{I}\right)$, where

$$
\sigma_{z_{1}}^{2}=\frac{\left(\Pi_{\mathbf{p}}^{j}-\Pi_{\mathbf{p}}^{k}\right)^{H} \Theta^{H} \Sigma_{c} \Theta\left(\Pi_{\mathbf{p}}^{j}-\Pi_{\mathbf{p}}^{k}\right)}{2 \sigma_{R}^{2}}
$$

and

$$
\sigma_{z_{2}}^{2}=\frac{\left(\Pi_{\mathbf{p}}^{j}+\Pi_{\mathbf{p}}^{k}-2 \Pi_{\mathbf{p}}^{i}\right)^{H} \Theta^{H} \Sigma_{c} \Theta\left(\Pi_{\mathbf{p}}^{j}+\Pi_{\mathbf{p}}^{k}-2 \Pi_{\mathbf{p}}^{i}\right)}{2 \sigma_{R}^{2}} .
$$

${ }^{1}$ For Rayleigh channel, $\mathbf{H}^{H} \mathbf{H}$ exactly follows the central complex Wishart distribution associated with $\Sigma_{c}=\mathbf{I}$
Also note that $\mathbb{E}\left\{\mathbf{z}_{1} \mathbf{z}_{2}^{H}\right\}=q \mathbf{I}$ and $\mathbb{E}\left\{\mathbf{z}_{2} \mathbf{z}_{1}^{H}\right\}=q^{H} \mathbf{I}$, where

$$
\begin{aligned}
q & =\frac{1}{2 \sigma_{R}^{2}} \operatorname{Tr}\left\{\left(\Pi_{\mathbf{p}}^{j}-\Pi_{\mathbf{p}}^{k}\right)\left(\Pi_{\mathbf{p}}^{j}+\Pi_{\mathbf{p}}^{k}-2 \Pi_{\mathbf{p}}^{i}\right)^{H} \Theta^{H} \Sigma_{c} \Theta\right\} \\
& =\frac{\left(\Pi_{\mathbf{p}}^{j}+\Pi_{\mathbf{p}}^{k}-2 \Pi_{\mathbf{p}}^{i}\right)^{H} \Theta^{H} \Sigma_{c} \Theta\left(\Pi_{\mathbf{p}}^{j}-\Pi_{\mathbf{p}}^{k}\right)}{2 \sigma_{R}^{2}} .
\end{aligned}
$$

Now we can rewrite $\gamma_{k j}$ in (45) as:

$$
\begin{aligned}
\gamma_{k j} \approx & \frac{1}{2 \sigma_{R}^{2}}\left(\left(\Pi_{\mathbf{p}}^{j}-\Pi_{\mathbf{p}}^{i}\right)-\left(\Pi_{\mathbf{p}}^{k}-\Pi_{\mathbf{p}}^{i}\right)\right)^{H} \mathbf{A}^{H} \mathbf{A} \\
& \left(\left(\Pi_{\mathbf{p}}^{j}-\Pi_{\mathbf{p}}^{i}\right)-\left(\Pi_{\mathbf{p}}^{k}-\Pi_{\mathbf{p}}^{i}\right)\right) \\
& =\left\|\mathbf{z}_{1}\right\|^{2} .
\end{aligned}
$$

Similarly $\kappa \approx \mathcal{R} e\left\{\frac{\mathbf{z}_{1}^{H} \mathbf{z}_{2}}{\left\|\mathbf{z}_{1}\right\|}\right\}$. First we derive the distribution of $\kappa$ given $\mathbf{z}_{1}$. Note that $\mathbf{z}_{1}$ and $\mathbf{z}_{2}$ are complex Gaussian distributed random vectors. Hence, the distribution $f_{\kappa \mid \mathbf{z}_{1}}\left(\kappa \mid \mathbf{z}_{1}\right)$ is Gaussian. Let $\tilde{\kappa}=\frac{\mathbf{z}_{1}^{H} \mathbf{z}_{2}}{\left\|\mathbf{z}_{1}\right\|}$. Therefore, using [79, prop. 3.13], we have $\mu_{\tilde{\kappa}}=$ $\mathbb{E}\left\{\tilde{\kappa} \mid \mathbf{z}_{1}=\tilde{\mathbf{z}}\right\}=\frac{q^{H}}{\sigma_{z_{1}}^{2}}\|\tilde{\mathbf{z}}\|$. Note that $\kappa=\mathcal{R} e\{\tilde{\kappa}\}$ and hence $\mathbb{E}\left\{\kappa \mid \mathbf{z}_{1}=\tilde{\mathbf{z}}\right\}=\mathcal{R} e\left\{\mu_{\tilde{\kappa}}\right\}=\frac{q_{R}}{\sigma_{z_{1}}^{2}}\|\tilde{\mathbf{z}}\|$, where $q_{R}=\mathcal{R} e\{q\}$. In order to compute variance of the $\kappa$ given $\mathbf{z}_{1}$, let us expand $\kappa$ as:

$\kappa=\mathcal{R} e\left\{\frac{\mathbf{z}_{1}^{H} \mathbf{z}_{2}}{\left\|\mathbf{z}_{1}\right\|}\right\}=\frac{\mathcal{R} e\left\{\mathbf{z}_{1}\right\}^{T} \mathcal{R} e\left\{\mathbf{z}_{2}\right\}}{\left\|\mathbf{z}_{1}\right\|}+\frac{\operatorname{I} m\left\{\mathbf{z}_{1}\right\}^{T} \operatorname{I} m\left\{\mathbf{z}_{2}\right\}}{\left\|\mathbf{z}_{1}\right\|}$

Let $u_{1}=\frac{\operatorname{Re}\left\{\mathbf{z}_{1}\right\}^{T} \operatorname{Re}\left\{\mathbf{z}_{2}\right\}}{\left\|\mathbf{z}_{1}\right\|}$ and $u_{2}=\frac{\operatorname{Im}\left\{\mathbf{z}_{1}\right\}^{T} \operatorname{Im}\left\{\mathbf{z}_{2}\right\}}{\left\|\mathbf{z}_{1}\right\|}$. Hence,

$$
\begin{aligned}
& \operatorname{var}\left(\kappa \mid \mathbf{z}_{1}=\tilde{\mathbf{z}}\right)=\operatorname{var}\left(u_{1} \mid \mathbf{z}_{1}=\tilde{\mathbf{z}}\right)+\operatorname{var}\left(u_{2} \mid \mathbf{z}_{1}=\tilde{\mathbf{z}}\right) \\
& +\operatorname{Cov}\left(u_{1}, u_{2} \mid \mathbf{z}_{1}=\tilde{\mathbf{z}}\right)+\operatorname{Cov}\left(u_{2}, u_{1} \mid \mathbf{z}_{1}=\tilde{\mathbf{z}}\right)
\end{aligned}
$$

Using [79, prop. 3.13], it can be shown that:

$$
\begin{aligned}
\operatorname{var}\left(u_{1} \mid \mathbf{z}_{1}=\tilde{\mathbf{z}}\right) & =\frac{\mathcal{R} e\{\tilde{\mathbf{z}}\}^{T} \operatorname{Cov}\left\{\mathcal{R} e\left\{\mathbf{z}_{2}\right\} \mid \mathbf{z}_{1}=\tilde{\mathbf{z}}\right\} \mathcal{R} e\{\tilde{\mathbf{z}}\}}{\|\tilde{\mathbf{z}}\|^{2}} \\
& =\frac{\mathcal{R} e\{\tilde{\mathbf{z}}\}^{2}}{\|\tilde{\mathbf{z}}\|^{2}} \frac{1}{2}\left(\sigma_{z_{2}}^{2}-\frac{\|q\|^{2}}{2 \sigma_{z_{1}}^{2}}\right)
\end{aligned}
$$

Similarly, $\operatorname{var}\left(u_{2} \mid \mathbf{z}_{1}=\tilde{\mathbf{z}}\right)=\frac{\operatorname{Im}\{\tilde{\mathbf{z}}\}^{2}}{\|\tilde{\mathbf{z}}\|^{2}} \frac{1}{2}\left(\sigma_{z_{2}}^{2}-\frac{\|q\|^{2}}{2 \sigma_{z_{1}}^{2}}\right)$ and

$$
\begin{aligned}
\operatorname{Cov}\left(u_{1}, u_{2} \mid \mathbf{z}_{1}=\tilde{\mathbf{z}}\right) & =-\operatorname{Cov}\left(u_{2}, u_{1} \mid \mathbf{z}_{1}=\tilde{\mathbf{z}}\right) \\
& =-j \frac{\|q\|^{2}}{4 \sigma_{z_{1}}^{2}} \frac{\operatorname{Re}\left\{\mathbf{z}_{1}\right\}^{T} \operatorname{I} m\left\{\mathbf{z}_{1}\right\}}{\left\|\mathbf{z}_{1}\right\|^{2}}
\end{aligned}
$$

Therefore, $\operatorname{var}\left(\kappa \mid \mathbf{z}_{1}=\tilde{\mathbf{z}}\right)=\frac{1}{2}\left(\sigma_{z_{2}}^{2}-\frac{\|q\|^{2}}{2 \sigma_{z_{1}}^{2}}\right)$. Hence, $\kappa \mid \mathbf{z}_{1} \sim \mathcal{N}\left(\frac{q_{R}}{\sigma_{z_{1}}^{2}}\|\tilde{\mathbf{z}}\|, \frac{\sigma_{\kappa}^{2}}{2}\right)$, where

$$
\sigma_{\kappa}^{2}=\left(\sigma_{z_{2}}^{2}-\frac{\|q\|^{2}}{2 \sigma_{z_{1}}^{2}}\right) \text {. }
$$


If $q_{R}=0, f_{K \mid \mathbf{z}_{1}}$ is independent of $\mathbf{z}_{1}$ and hence the unconditional distribution of $\kappa$ is the same as the conditional distribution, i.e., $\kappa \sim \mathcal{N}\left(0, \frac{\sigma_{\kappa}^{2}}{2}\right)$.

When $q_{R} \neq 0$, the unconditional distribution can be obtained by eliminating the conditioning with respect to the distribution of $\|\tilde{z}\|$, which is Nakagami distributed Nakagami $\left(N_{R}, N_{R} \sigma_{z_{1}}^{2}\right)$, since $\|\tilde{z}\|^{2} \sim$ $\Gamma\left(N_{R}, \frac{1}{\sigma_{z_{1}}^{2}}\right)$. Explicitly, $f_{\bar{Z}}(\bar{z})=\frac{2 \bar{z}^{2 N_{R}-1} e^{-\frac{\bar{z}^{2}}{\sigma_{z_{1}}^{2}}}}{\Gamma\left(N_{R}\right)\left(\sigma_{z_{1}}^{2}\right)^{N_{R}}}[80]$, where $\bar{z}=\|\tilde{z}\|$. Hence, the unconditional distribution of $\kappa$ is:

$$
f_{\kappa}(\kappa)=\frac{2 \int_{0}^{\infty} e^{-g(\kappa, \bar{z})} \bar{z}^{2 N_{R}-1} d \bar{z}}{\left(\sigma_{z_{1}}^{2}\right)^{N_{R}} \Gamma\left(N_{R}\right) \sqrt{\left(\pi \sigma_{\kappa}^{2}\right)}}
$$

where

$$
\begin{aligned}
g(\kappa, \bar{z}) & =\frac{\left(\kappa-\frac{q_{R}}{\sigma_{z_{1}}^{2}} \bar{z}\right)^{2}}{\sigma_{\kappa}^{2}}+\frac{\bar{z}^{2}}{\sigma_{z_{1}}^{2}} \\
& =\frac{\kappa^{2}}{\sigma_{\kappa}^{2}}+\frac{q_{R}^{2}+\sigma_{z_{1}}^{2} \sigma_{\kappa}^{2}}{\left(\sigma_{z_{1}}^{2}\right)^{2} \sigma_{\kappa}^{2}} \bar{z}^{2}-\frac{2 q_{R} \kappa}{\sigma_{z_{1}}^{2} \sigma_{\kappa}^{2}} \bar{z} .
\end{aligned}
$$

Now using [72, 3.462(1)], we arrive at:

$$
\begin{aligned}
I_{\kappa} & =\int_{0}^{\infty} e^{-g(\kappa, \bar{z})} \bar{z}^{2 N_{R}-1} d \bar{z} \\
& =e^{-\frac{\kappa^{2}}{\sigma_{\kappa}^{2}}} \int_{0}^{\infty} e^{-\frac{q_{R}^{2}+\sigma_{z_{1}}^{2} \sigma_{\kappa}^{2}}{\left(\sigma_{z_{1}}^{2}\right)^{2} \sigma_{\kappa}^{2}} \bar{z}^{2}+\frac{2 q_{R} \kappa}{\sigma_{z_{1}}^{2} \sigma_{\kappa}^{2}} \bar{z}} \bar{z}^{2 N_{R}-1} d \bar{z} \\
& =e^{-\frac{\kappa^{2}}{\sigma_{\kappa}^{2}}}\left(\frac{\sigma_{z_{1}}^{2}(v-1)}{2 v}\right)^{N_{R}} \Gamma\left(2 N_{R}\right) e^{\frac{\kappa^{2}}{2 \sigma_{\kappa}^{2} v}} D_{-2 N_{R}}\left(-\sqrt{\frac{2}{\sigma_{K}^{2} v} \kappa}\right),
\end{aligned}
$$

where $v=1+\frac{\sigma_{\kappa}^{2} \sigma_{z_{1}}^{2}}{q_{R}^{2}}$. Finally, substituting (57) into (55) will give (44).

\section{REFERENCES}

[1] F. Tariq, M. Khandaker, K.-K. Wong, M. Imran, M. Bennis, and M. Debbah, "A speculative study on 6G," arXiv preprint arXiv:1902.06700, 2019.

[2] S. Rangan, T. S. Rappaport, and E. Erkip, "Millimeter-wave cellular wireless networks: Potentials and challenges," Proceedings of the IEEE, vol. 102, pp. 366-385, March 2014.

[3] T. S. Rappaport, S. Sun, R. Mayzus, H. Zhao, Y. Azar, K. Wang, G. N. Wong, J. K. Schulz, M. Samimi, and F. Gutierrez, "Millimeter Wave Mobile Communications for 5G Cellular: It Will Work!," IEEE Access, vol. 1, pp. 335349, 2013.

[4] T. S. Rappaport, "Spectrum frontiers: The new world of millimeter-wave mobile communication," Invited keynote presentation, The Federal Communications Commission (FCC) Headquarters, vol. 10, 2016.

[5] "Part 11: Wireless LAN medium access control (MAC) and physical layer (PHY) specifications amendment 3: enhancements for very high throughput in the $60 \mathrm{GHz}$ band," IEEE Std 802.11ad-2012 (Amendment to IEEE Std 802.11-2012, as amended by IEEE Std 802.11ae-2012 and IEEE Std 802.11aa2012), pp. 1-628, Dec 2012.

[6] W. Roh, J. Seol, J. Park, B. Lee, J. Lee, Y. Kim, J. Cho, K. Cheun, and F. Aryanfar, "Millimeter-wave beamforming as an enabling technology for $5 \mathrm{G}$ cellular communications: theoretical feasibility and prototype results," IEEE Communications Magazine, vol. 52, pp. 106-113, February 2014.
[7] T. S. Rappaport, Y. Xing, G. R. MacCartney, A. F. Molisch, E. Mellios, and J. Zhang, "Overview of millimeter wave communications for fifth-generation (5G) wireless networkswith a focus on propagation models," IEEE Transactions on Antennas and Propagation, vol. 65, pp. 6213-6230, Dec 2017.

[8] A. I. Sulyman, A. Alwarafy, G. R. MacCartney, T. S. Rappaport, and A. Alsanie, "Directional radio propagation path loss models for millimeter-wave wireless networks in the 28, 60-, and 73-GHz bands," IEEE Transactions on Wireless Communications, vol. 15, pp. 6939-6947, Oct 2016.

[9] L. Dai, B. Wang, M. Wang, X. Yang, J. Tan, S. Bi, S. Xu, F. Yang, Z. Chen, M. Di Renzo, et al., "Reconfigurable intelligent surface-based wireless communication: Antenna design, prototyping and experimental results," arXiv preprint arXiv:1912.03620, 2019.

[10] W. Qingqing and Z. Rui, "Towards smart and reconfigurable environment: Intelligent reflecting surface aided wireless network," arXiv preprint arXiv:1905.00152, 2019.

[11] M. Di Renzo, M. Debbah, D.-T. Phan-Huy, A. Zappone, M.-S. Alouini, C. Yuen, V. Sciancalepore, G. C. Alexandropoulos, J. Hoydis, H. Gacanin, et al., "Smart radio environments empowered by reconfigurable AI meta-surfaces: an idea whose time has come," EURASIP Journal on Wireless Communications and Networking, vol. 2019, no. 1, pp. 1-20, 2019.

[12] C. Huang, S. Hu, G. C. Alexandropoulos, A. Zappone, C. Yuen, R. Zhang, M. Di Renzo, and M. Debbah, "Holographic MIMO surfaces for 6G wireless networks: Opportunities, challenges, and trends," arXiv preprint arXiv:1911.12296, 2019.

[13] L. Subrt and P. Pechac, "Controlling propagation environments using intelligent walls," in 2012 6th European Conference on Antennas and Propagation (EUCAP), pp. 1-5, March 2012.

[14] X. Tan, Z. Sun, J. M. Jornet, and D. Pados, "Increasing indoor spectrum sharing capacity using smart reflect-array," in 2016 IEEE International Conference on Communications (ICC), pp. 1-6, May 2016

[15] W. Yan, X. Kuai, X. Yuan, et al., "Passive beamforming and information transfer via large intelligent surface," arXiv preprint arXiv:1905.01491, 2019.

[16] X. Yu, D. Xu, and R. Schober, "MISO wireless communication systems via intelligent reflecting surfaces : (Invited paper)," in 2019 IEEE/CIC International Conference on Communications in China (ICCC), pp. 735-740, Aug 2019.

[17] Y. Han, W. Tang, S. Jin, C. Wen, and X. Ma, "Large intelligent surface-assisted wireless communication exploiting statistical CSI," IEEE Transactions on Vehicular Technology, vol. 68, pp. 8238-8242, Aug 2019.

[18] C. Huang, A. Zappone, G. C. Alexandropoulos, M. Debbah, and C. Yuen, "Reconfigurable intelligent surfaces for energy efficiency in wireless communication," IEEE Transactions on Wireless Communications, vol. 18, pp. 4157-4170, Aug 2019.

[19] C. Huang, G. C. Alexandropoulos, A. Zappone, M. Debbah, and C. Yuen, "Energy efficient multi-user MISO communication using low resolution large intelligent surfaces," in 2018 IEEE Globecom Workshops (GC Wkshps), pp. 1-6, IEEE, 2018.

[20] Q. Wu and R. Zhang, "Intelligent reflecting surface enhanced wireless network via joint active and passive beamforming," IEEE Transactions on Wireless Communications, vol. 18, pp. 5394-5409, Nov 2019.

[21] J. Chen, Y. Liang, Y. Pei, and H. Guo, "Intelligent reflecting surface: A programmable wireless environment for physical layer security," IEEE Access, vol. 7, pp. 82599-82612, 2019.

[22] C. Pan, H. Ren, K. Wang, W. Xu, M. Elkashlan, A. Nallanathan, and L. Hanzo, "Multicell MIMO communications relying on intelligent reflecting surface," Online] https://arxiv. org/abs/1907.10864, 2019.

[23] C. Pan, H. Ren, K. Wang, M. Elkashlan, A. Nallanathan, J. Wang, and L. Hanzo, "Intelligent reflecting surface enhanced MIMO broadcasting for simultaneous wireless information and power transfer," arXiv preprint arXiv:1908.04863, 2019.

[24] T. Jiang and Y. Shi, "Over-the-air computation via intelligent reflecting surfaces," arXiv preprint arXiv:1904.12475, 2019.

[25] T. Bai, C. Pan, Y. Deng, M. Elkashlan, A. Nallanathan, and L. Hanzo, "Latency minimization for intelligent reflecting surface aided mobile edge computing," arXiv preprint arXiv:1910.07990, 2019. 
[26] E. Basar, "Reconfigurable intelligent surface-based index modulation: A new beyond MIMO paradigm for 6G," IEEE Transactions on Communications, vol. 68, no. 5, pp. 3187-3196, 2020.

[27] Y. Ding, V. Fusco, A. Shitvov, Y. Xiao, and H. Li, "Beam index modulation wireless communication with analog beamforming," IEEE Transactions on Vehicular Technology, vol. 67, pp. 6340-6354, July 2018.

[28] R. Y. Mesleh, H. Haas, S. Sinanovic, C. W. Ahn, and S. Yun "Spatial modulation," IEEE Transactions on Vehicular Technology, vol. 57, pp. 2228-2241, July 2008.

[29] P. Yang, M. Di Renzo, Y. Xiao, S. Li, and L. Hanzo, "Design guidelines for spatial modulation," IEEE Communications Surveys Tutorials, vol. 17, pp. 6-26, Firstquarter 2015.

[30] S. Kutty and D. Sen, "Beamforming for millimeter wave communications: An inclusive survey," IEEE Communications Surveys Tutorials, vol. 18, pp. 949-973, Secondquarter 2016.

[31] Z. Xiao, X. Xia, D. Jin, and N. Ge, "Iterative eigenvalue decomposition and multipath-grouping $\mathrm{Tx} / \mathrm{Rx}$ joint beamformings for millimeter-wave communications," IEEE Transactions on Wireless Communications, vol. 14, pp. 1595-1607, March 2015.

[32] O. Jo, W. Hong, S. T. Choi, S. Chang, C. Kweon, J. Oh, and K. Cheun, "Holistic design considerations for environmentally adaptive $60 \mathrm{GHz}$ beamforming technology," IEEE Communications Magazine, vol. 52, pp. 30-38, Nov 2014

[33] Y. Li, J. Luo, M. H. CastaÃśeda Garcia, R. BÃúhnke, R. A. Stirling-Gallacher, W. Xu, and G. Caire, "On the beamformed broadcasting for millimeter wave cell discovery: Performance analysis and design insight," IEEE Transactions on Wireles. Communications, vol. 17, pp. 7620-7634, Nov 2018.

[34] Z. Xiao, T. He, P. Xia, and X. Xia, "Hierarchical codebook design for beamforming training in millimeter-wave communication," IEEE Transactions on Wireless Communications, vol. 15, pp. 3380-3392, May 2016.

[35] I. Aykin and M. Krunz, "Efficient beam sweeping algorithms and initial access protocols for millimeter-wave networks," IEEE Transactions on Wireless Communications, pp. 1-1, 2020.

[36] Z. Sha, Z. Wang, S. Chen, and L. Hanzo, "Graph theory based beam scheduling for inter-cell interference avoidance in mmwave cellular networks," IEEE Transactions on Vehicular Technology, pp. 1-1, 2020.

[37] Y. Ju, H. Wang, T. Zheng, Q. Yin, and M. H. Lee, "Safeguarding millimeter wave communications against randomly located eavesdroppers," IEEE Transactions on Wireless Communications, vol. 17, pp. 2675-2689, April 2018.

[38] A. E. Canbilen, E. Basar, and S. S. Ikki, "Reconfigurable intelligent surface-assisted space shift keying," IEEE Wireless Communications Letters, 2020.

[39] K. Ying, Z. Gao, S. Lyu, Y. Wu, H. Wang, and M. Alouini, "GMD-based hybrid beamforming for large reconfigurable intelligent surface assisted millimeter-wave massive MIMO," IEEE Access, vol. 8, pp. 19530-19539, 2020

[40] X. Yang, C.-K. Wen, and S. Jin, "MIMO detection for reconfigurable intelligent surface-assisted millimeter wave systems,' arXiv preprint arXiv:2004.06001, 2020.

[41] B. Sadhu, Y. Tousi, J. Hallin, S. Sahl, S. Reynolds, O. Renstrom, K. Sjogren, O. Haapalahti, N. Mazor, B. Bokinge G. Weibull, H. Bengtsson, A. Carlinger, E. Westesson, J. Thillberg, L. Rexberg, M. Yeck, X. Gu, D. Friedman, and A. ValdesGarcia, "7.2 A 28GHz 32-element phased-array transceiver IC with concurrent dual polarized beams and 1.4 degree beamsteering resolution for 5G communication," in 2017 IEEE International Solid-State Circuits Conference (ISSCC), pp. 128 129, Feb 2017.

[42] C. Scarborough, K. Venugopal, A. Alkhateeb, and R. W. Heath "Beamforming in millimeter wave systems: Prototyping and measurement results," in 2018 IEEE 88th Vehicular Technology Conference (VTC-Fall), pp. 1-5, Aug 2018.

[43] S. Han, C. I, Z. Xu, and C. Rowell, "Large-scale antenna systems with hybrid analog and digital beamforming for millimeter wave 5G," IEEE Communications Magazine, vol. 53, pp. 186-194, January 2015.

[44] J. Zhang, Y. Huang, J. Wang, R. Schober, and L. Yang, "Powerefficient beam designs for millimeter wave communication systems," IEEE Transactions on Wireless Communications, vol. 19, pp. 1265-1279, Feb 2020.

[45] R. Rajashekar and L. Hanzo, "Hybrid beamforming in mmwave MIMO systems having a finite input alphabet," IEEE Transactions on Communications, vol. 64, pp. 3337-3349, Aug 2016.

[46] A. M. Elbir and K. V. Mishra, "Joint antenna selection and hybrid beamformer design using unquantized and quantized deep learning networks," IEEE Transactions on Wireless Communications, pp. 1-1, 2019.

[47] C. Zhao, Y. Cai, A. Liu, M. Zhao, and L. Hanzo, "Mobile edge computing meets mmwave communications: Joint beamforming and resource allocation for system delay minimization," IEEE Transactions on Wireless Communications, pp. 1-1, 2020.

[48] K. Satyanarayana, M. El-Hajjar, A. A. M. Mourad, and L. Hanzo, "Multi-user hybrid beamforming relying on learning-aided link-adaptation for mmwave systems," IEEE Access, vol. 7, pp. 23197-23209, 2019.

[49] S. Dutta, C. N. Barati, D. Ramirez, A. Dhananjay, J. F. Buckwalter, and S. Rangan, "A case for digital beamforming at mmwave," IEEE Transactions on Wireless Communications, vol. 19, pp. 756-770, Feb 2020

[50] W. T. Sethi, H. Vettikalladi, and M. A. Alkanhal, "Millimeter wave antenna with mounted horn integrated on FR4 for 60 GHz Gbps communication systems," International Journal of Antennas and Propagation, vol. 2013, 2013.

[51] G. Zhou, C. Pan, H. Ren, K. Wang, and A. Nallanathan, "A framework of robust transmission design for IRS-aided MISO communications with imperfect cascaded channels," arXiv preprint arXiv:2001.07054, 2020.

[52] E. Basar, U. Aygolu, E. Panayirci, and H. V. Poor, "Orthogonal frequency division multiplexing with index modulation," IEEE Transactions on Signal Processing, vol. 61, pp. 5536-5549, Nov 2013.

[53] S. Gopi, S. Kalyani, and L. Hanzo, "Coherent and non-coherent multilayer index modulation," IEEE Access, vol. 7, pp. 7967779693, 2019

[54] C. D. Meyer, Matrix analysis and applied linear algebra, vol. 71. SIAM, 2000.

[55] K. Deb, Optimization for engineering design: Algorithms and examples. PHI Learning Pvt. Ltd., 2012.

[56] M. R. Osborne and G. A. Watson, "An algorithm for minimax approximation in the nonlinear case," The Computer Journal, vol. 12 , no. 1 , pp. 63-68, 1969.

[57] H. Yang, X. Chen, F. Yang, S. Xu, X. Cao, M. Li, and J. Gao, "Design of resistor-loaded reflectarray elements for both amplitude and phase control," IEEE Antennas and Wireless Propagation Letters, vol. 16, pp. 1159-1162, 2017.

[58] H. L. Van Trees, Optimum array processing: Part IV of detection, estimation, and modulation theory. John Wiley \& Sons, 2004.

[59] C. A. Balanis, Antenna theory: analysis and design. John wiley \& sons, 2016

[60] Y. Pati, R. Rezaiifar, and P. Krishnaprasad, "Orthogonal matching pursuit: Recursive function approximation with applications to wavelet decomposition," in Conference Record of The Twenty-Seventh Asilomar Conference on Signals, Systems and Computers, 1993. , pp. 40-44 vol.1, Nov 1993.

[61] D. Needell and J. A. Tropp, "CoSaMP: Iterative signal recovery from incomplete and inaccurate samples," Applied and Computational Harmonic Analysis, vol. 26, no. 3, pp. 301-321, 2009.

[62] M. Fréchet, "Genéralisation du théoreme des probabilités totales," Fundamenta mathematicae, vol. 1, no. 25, pp. 379-387, 1935.

[63] Z. Wang, L. Liu, and S. Cui, "Channel estimation for intelligent reflecting surface assisted multiuser communications: Framework, algorithms, and analysis," arXiv preprint arXiv:1912.11783, 2019.

[64] D. Donoho, "Compressed sensing," IEEE Transactions on information theory, vol. 52, pp. 1289-1306, April 2006.

[65] Yuguang Fang, K. A. Loparo, and Xiangbo Feng, "Inequalities for the trace of matrix product," IEEE Transactions on Automatic Control, vol. 39, pp. 2489-2490, Dec 1994. 
[66] T. Eng and L. B. Milstein, "Coherent DS-CDMA performance in Nakagami multipath fading," IEEE Transactions on Communications, vol. 43, pp. 1134-1143, Feb 1995.

[67] A. M. Mathai and R. K. Saxena, Generalized hypergeometric functions with applications in statistics and physical sciences, vol. 348. Springer, 2006.

[68] H. Buchholz, The confluent hypergeometric function: with special emphasis on its applications, vol. 15. Springer Science \& Business Media, 2013

[69] J. G. Proakis and M. Salehi, Digital communications, vol. 4. McGraw-hill New York, 2001.

[70] Wolfram Research, "Complimentary error function." [Online]. Available: http://functions.wolfram.com/GammaBetaErf/ Erfc/26/01/01/. Last visited on 15/2/2020.

[71] P. Sebah and X. Gourdon, "Introduction to the Gamma function." [Online]. Available: https://www.csie.ntu.edu.tw/ w89089/link/gammaFunction.pdf. Last visited on 15/2/2020

[72] I. S. Gradshteyn and I. M. Ryzhik, Table of integrals, series, and products. Academic press, 2014.

[73] S. Kalyani, "On CRB for parameter estimation in two component gaussian mixtures and the impact of misspecification," IEEE Transactions on Communications, vol. 60, pp. 37343744, December 2012.

[74] Wolfram Research, "Gauss hypergeometric function ${ }_{2} F_{1}$." [Online]. Available: http://functions.wolfram.com/ HypergeometricFunctions/Hypergeometric2F1/16/01/01/. Last visited on $15 / 2 / 2020$.

[75] Wolfram Research, "Gauss hypergeometric function ${ }_{2} F_{1}$." [Online]. Available: http://functions.wolfram.com/ HypergeometricFunctions/Hypergeometric2F1/03/06/01/0006/. Last visited on 15/2/2020.

[76] Wolfram Research, "Incomplete beta function." [Online]. Available: http://functions.wolfram.com/GammaBetaErf/ Beta3/03/01/01/. Last visited on 15/2/2020.

[77] M. Geller and E. W. Ng, "A table of integrals of the error function.," Journal of Research of the National Bureau of Standards- B Mathematical Sciences, vol. 73B, no. 1, 1969.

[78] R. Xu, Z. Zhong, and J.-M. Chen, "Approximation to the capacity of rician fading mimo channels," in VTC Spring 2009 IEEE 69th Vehicular Technology Conference, pp. 1-5, IEEE, 2009.

[79] M. L. Eaton, "Multivariate statistics: a vector space approach.," John Wiley \& Sons, Inc., 605 Third Avenue., New York, NY 10158, USA, 1983, 512, 1983.

[80] M. Nakagami, "The m-distribution - A general formula of intensity distribution of rapid fading," in Statistical methods in radio wave propagation, pp. 3-36, Elsevier, 1960. 